\title{
Diverse Genetic Determinants of Nitrofurantoin Resistance in UK
}

Yu Wan ${ }^{1}$, Ewurabena Mills ${ }^{1,2}$, Rhoda C.Y. Leung ${ }^{1 *}$, Ana Vieira ${ }^{1,2}$, Elita Jauneikaite ${ }^{1,3,4}$, Xiangyun

Zhi $^{1,2}$, Nicholas J. Croucher ${ }^{3,4}$, Neil Woodford ${ }^{5}$, Matthew J. Ellington ${ }^{1,5}$, and Shiranee Sriskandan ${ }^{1,2 \#}$

${ }^{1}$ NIHR Health Protection Research Unit in Healthcare Associated Infections and Antimicrobial

${ }^{2}$ MRC Centre for Molecular Bacteriology and Infection, Imperial College London, London, UK

${ }^{3}$ Department of Infectious Disease Epidemiology, School of Public Health, Imperial College

${ }^{4}$ MRC Centre for Global Infectious Disease Analysis, School of Public Health, Imperial College

${ }^{5}$ Antimicrobial Resistance and Healthcare Associated Infections Reference Unit, National Infection 


\section{Abstract}

17 Antimicrobial resistance in enteric or urinary Escherichia coli is a risk factor for invasive E. coli

18 infections. Due to widespread trimethoprim resistance amongst urinary E. coli and increased

19 bacteraemia incidence, a national recommendation to prescribe nitrofurantoin for uncomplicated

20 urinary tract infection was made in 2018. Nitrofurantoin resistance is reported in $<6 \%$ urinary E. coli

21 isolates in the UK. However, mechanisms underpinning nitrofurantoin resistance in these isolates

22 remain unknown. This study aimed to identify genetic determinants of nitrofurantoin resistance in a

23 local E. coli collection and assess their prevalence in a larger dataset of $E$. coli genomes. Deleterious

24 point mutations and gene-inactivating insertion sequences in both chromosomal nitroreductase genes

$25 n f s A$ and $n f s B$ were identified in genomes of nine nitrofurantoin-resistant urinary $E$. coli isolates

26 collected from north west London. Eight types of genetic alterations were identified when comparing

27 sequences of $n f s A, n f s B$, and the associated gene ribE in 12,412 E. coli genomes collected from across the UK. Evolutionary analysis revealed homoplasic mutations and explained the order of stepwise mutations. An algorithm was developed to predict nitrofurantoin susceptibility and predictions for 20 accessible isolates were experimentally validated. Only one genome carrying oqxAB, a mobile gene complex associated with reduced nitrofurantoin susceptibility, was identified. In conclusion, mutations and insertion sequences in $n f_{s} A$ and $n f_{s} B$ are leading causes of nitrofurantoin resistance in UK $E$. coli. As nitrofurantoin exposure increases in human populations, the prevalence of nitrofurantoin resistance in carriage $E$. coli isolates and those from urinary and bloodstream infections should be monitored.

\section{Importance}

37 This study expands knowledge about the genetic basis of nitrofurantoin resistance in $E$. coli isolates using whole-genome sequencing and genomic analysis. We report novel and previously known deleterious mutations of chromosomal genes $n f s A, n f s B$, and $r i b E$ as well as the interruption of $n f s A$ and $n f s B$ by insertion sequences, recapitulating the roles of oxygen-insensitive nitroreductases in the 
41 development of nitrofurantoin resistance in E. coli. We revealed and categorised the genotypic

42 diversity in these three genes in a large collection of UK E. coli genomes. A scoring algorithm is

43 provided to predict nitrofurantoin susceptibility from genotypes. Our predictions suggest that acquired

44 nitrofurantoin resistance is not of immediate concern in the UK. However, experimental validation of

45 predictions suggested the involvement of mechanisms other than alterations in $n f s A$, $n f s B$, or $\operatorname{ribE}$ in

46 determining nitrofurantoin susceptibility, emphasising the need for monitoring nitrofurantoin

47 resistance amongst E. coli.

48

49 Keywords Escherichia coli, nitrofurantoin, antibiotic resistance, bacteraemia, urinary tract infection,

50 nitroreductases, resistance mechanisms, mutations, insertion sequences, predicting antimicrobial

51 resistance 


\section{Introduction}

53 Nitrofurantoin is a synthetic nitrofuran compound that has been widely used as a first-line

54 antimicrobial agent for treating urinary tract infection (UTI) since 1953 and is active against a broad

55 range of Gram-negative and Gram-positive bacteria, including Escherichia coli, most species of

56 Staphylococcus and Enterococcus $(1,2)$. It can reach a urinary concentration of 50-250 mg/L while

57 retaining negligible blood concentration at standard therapeutic dosage, providing an advantage for

58 treating UTI $(3,4)$. Although the antibacterial activity of nitrofurantoin is not fully understood, studies

59 have revealed that metabolic intermediates from nitroreductase-mediated reduction of nitrofurantoin

60 can damage DNA and RNA, as well as disrupting protein production (5-7).

E. coli is the predominant pathogen of uncomplicated UTI, and the increasing prevalence of isolates

resistant to antimicrobials such as beta-lactams, ampicillin, and trimethoprim $(8,9)$ has led the UK

63 National Institute for Health and Care Excellence (NICE, www.nice.org.uk) to recommend prescribing

64 nitrofurantoin for UTI since November 2018 (10). Despite clinical use of nitrofurantoin for nearly 70

65 years, the prevalence of nitrofurantoin resistance in E. coli remains relatively low in Europe. Up to

2016, fewer than $6 \%$ of E. coli isolates collected from urine specimens in Western, Northern, and

Southern European countries were resistant to nitrofurantoin (11-15). Separate UK-based studies showed that nitrofurantoin-resistant $E$. coli accounted for $5 \%$ of urinary and bloodstream E. coli

69 isolates collected in London, during 2005-2006 and 2011-2015, despite higher prevalence of resistance (>20\%) to other commonly prescribed oral antimicrobials in the same isolates (16-18).

Since E. coli was not widely exposed to nitrofurantoin in the UK until adoption of the new NICE guideline, it may be too early to detect any trend in the prevalence of nitrofurantoin resistance among

73 UK E. coli isolates. The reported low prevalence of nitrofurantoin resistance might be explained by

74 the broad intracellular target range of toxic nitrofurantoin metabolites and a reported fitness cost

75 among nitrofurantoin-resistant mutants $(5,19)$. Two kinds of genetic determinants of nitrofurantoin 
chromosomal genes $n f s A, n f s B$ (encoding oxygen-insensitive nitroreductases NfsA and NfsB, respectively), and ribE (encoding 6,7-dimethyl-8-ribityllumazine synthase, involved in the reduction of nitrofurantoin) $(6,7)$; and (b) acquired gene complex $o q x A B$ (encoding a multidrug efflux pump OqxAB) (20). By contrast, bacteria with impaired DNA-repair ability showed increased susceptibility

81 to nitrofurantoin $(21,22)$.

82 Understanding the mechanisms underpinning antimicrobial resistance (AMR) is central to 83 predicting the impact of antimicrobial prescribing guidelines. Nevertheless, there is little or no 84 understanding of the basis of nitrofurantoin resistance in UK E. coli. This is hindered by the fact that 85 nitrofurantoin susceptibility testing is mostly limited to urinary tract isolates rather than enteric or 86 invasive bloodstream isolates, while a vast array of whole-genome sequencing (WGS) has focussed 87 on bloodstream isolates or isolates with multidrug-resistance phenotypes. We set out to uncover the 88 genetic basis of nitrofurantoin resistance in E. coli isolated from UTI patients in north west London.

89 We then screened a large genomic dataset of 12,412 UK E. coli isolates for resistance-associated 90 genetic alterations to enhance our understanding of the wider distribution of nitrofurantoin resistance 91 and genetic mechanisms that influence nitrofurantoin's interaction with E. coli.

\section{Results}

\section{Clinical $E$. coli isolates from north west London}

94 Nitrofurantoin resistance (MIC $>64 \mathrm{mg} / \mathrm{L}$ ) was confirmed in nine out of 18 E. coli UTI isolates that 95 had been reported as nitrofurantoin-resistant by an NHS diagnostic laboratory (supplementary Table 96 S1), following protocols and clinical breakpoints specified by the European Committee on 97 Antimicrobial Susceptibility Testing (EUCAST). Only one isolate was obtained from each patient. 98 Genomic DNA from these nine isolates (IN01-09) was subject to WGS and compared with genomic 99 sequences of E. coli with known nitrofurantoin susceptibility. 


\section{Population structure of $\boldsymbol{E}$. coli isolates with known nitrofurantoin susceptibility}

101 To contextualise the isolates IN01-09, genomes of additional 208 E. coli isolates with known 102 nitrofurantoin-susceptibility phenotypes were incorporated into analysis (Table S1). Of these, eight 103 isolates were nitrofurantoin-resistant, none of which came from the UK, and 200 isolates were 104 nitrofurantoin-susceptible, 14 of which came from the UK.

105 Population structure of the 217 isolates (including IN01-09) was interpreted using both multi-locus 106 sequence types (MLSTs) and genomic similarities. In total, 57 E. coli sequence types (STs) 107 constituting 19 clonal complexes (CCs, defined by the MLST scheme) were identified (Fig. 1 and 108 Table S1). No novel MLST allele or ST was detected. Isolates IN01-09 were classified into seven STs 109 (Table 1). The 217 E. coli isolates were further grouped into 38 genomic clusters using PopPUNK (23), 110 and as expected, these clusters showed a high congruence with CCs (Fig. 1). Notably, isolates IN01 111 and IN02 only slightly differed in presence-absence of accessory genes by a PopPUNK accessory112 genome distance (Jaccard distance) of $2.4 \times 10^{-4}$, although they were collected from different patients 113 with distinct community UTI episodes. Interestingly, while the majority of nitrofurantoin-resistant

114 isolates were sporadically distributed across these CCs (including major clinical lineages such as CC69, 115 CC73, and CC131), clustering of reduced nitrofurantoin susceptibility was seen among USA isolates of CC38 (Fig. 1 and Table S1). 


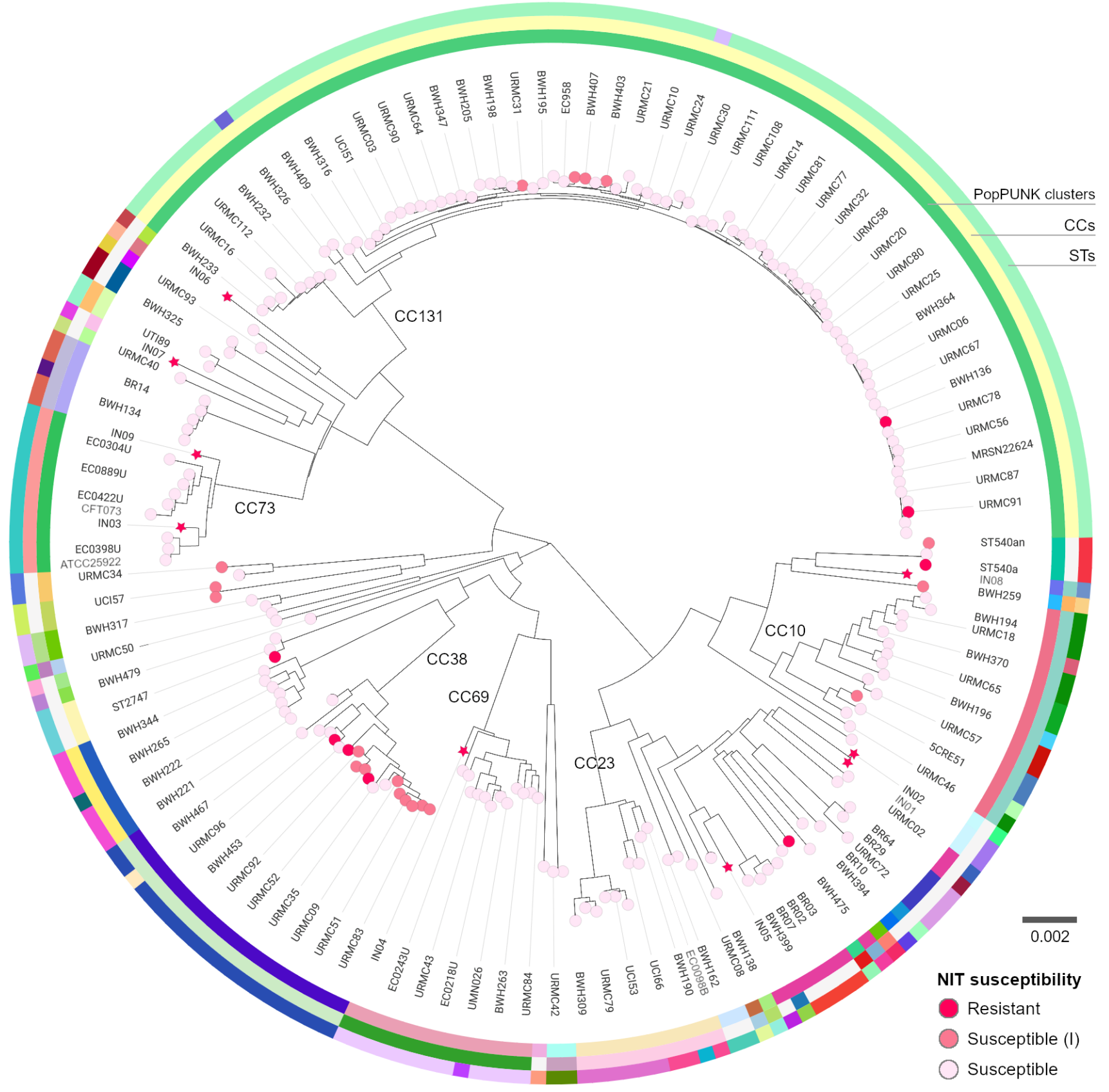

FIG 1 Population structure of 217 E. coli isolates with known nitrofurantoin (NIT) susceptibility.

119 Relatedness between these isolates is displayed by a core-genome neighbour-joining tree generated

120 using PopPUNK. The scale bar denotes the density of core-genome single-nucleotide polymorphisms

121 per unit of branch lengths (23). The tree is midpoint-rooted and drawn in a circular layout with tips

122 coloured by nitrofurantoin susceptibility of isolates (Table S1). Stars highlight isolates IN01-09. CCs

123 having $\geq 10$ genomes are labelled over the tree. Following EUCAST guidelines, an isolate was

124 considered susceptible under an increased exposure of nitrofurantoin, denoted as "susceptible (I)", 
125 when $32 \mathrm{mg} / \mathrm{L}<\mathrm{MIC} \leq 64 \mathrm{mg} / \mathrm{L}$. Full data from this figure can be interactively visualised on

Microreact (microreact.org/project/pHwAhGZ55AL7CvXxGnziyU) (24).

\section{Genetic basis of nitrofurantoin resistance in UK $E$. coli isolates IN01-09}

128 Isolates IN01-09 showed a minimum nitrofurantoin MIC of $128 \mathrm{mg} / \mathrm{L}$, and the MICs of three isolates

129 exceeded $512 \mathrm{mg} / \mathrm{L}$ (Table 1). No public WGS data of other nitrofurantoin-resistant UK E. coli isolates

130 was found as of May 2020. We focused on alternations in intrinsic genes $n f s A, n f s B$, ribE, and 131 presence-absence of the acquired gene complex $o q x A B$. For each genome of isolates IN01-09, its most 132 closely related complete genome (based on PopPUNK core-genome distances) of a nitrofurantoin133 susceptible E. coli isolate was chosen as a reference for the identification of genetic alterations.

\section{Genetic alterations of $n f s A$ and $n f s B$}

135 Analysis of $n f s A$ and $n f s B$ confirmed the presence of a single copy of each gene in each of the nine 136 genomes IN01-09 (Table S2). In each of these genomes, genetic alterations affecting protein 137 sequences were identified in both $n f s A$ and $n f s B$ when alleles of both genes were compared with those 138 in the reference genome. Altogether, these alterations consisted of missense and nonsense mutations, 139 deletions, and interruptions by insertion sequences (Table 1). Correspondingly, predicted sequences of 140 proteins NfsA and NfsB showed amino acid substitutions or truncations or both. Synonymous 141 mutations in $n f s A$ or $n f s B$ were also detected in five genomes (Table S2). One deleterious missense 142 mutation was predicted by PROVEAN (25) for each NfsA or NfsB sequence that only carried missense 143 mutations (Table 1). These deleterious missense mutations were not present in any NfsA or NfsB 144 sequence from the other $208 \mathrm{E}$. coli isolates whose nitrofurantoin susceptibility was known. Only two 145 deleterious missense mutations (W212R in NfsA and G192D in NfsB) were previously reported as 146 associated with nitrofurantoin resistance (appendix Tables A1 and A2). 
148 TABLE 1 Nonsynonymous mutations and gene interruptions in $n f s A$ and $n f s B$ of isolates IN01-09.

\begin{tabular}{|c|c|c|c|c|c|c|c|}
\hline Isolate & $\begin{array}{c}\text { MIC } \\
(\mathrm{mg} / \mathrm{L})\end{array}$ & ST & Reference & Gene & $\begin{array}{l}\text { Nucleotide } \\
\text { change }\end{array}$ & $\begin{array}{l}\text { Protein } \\
\text { change }\end{array}$ & $\begin{array}{c}\text { PROVEAN } \\
\text { score }\end{array}$ \\
\hline \multirow[t]{2}{*}{ IN01 } & 256 & 1463 & NZ_CP035320.1 & $n f_{S A}$ & 693InsIS1R & Interruption & NA \\
\hline & & & (BR02) & $n f_{s} B$ & $575 \mathrm{G}>\mathrm{A}$ & G192D & $-6.954 *$ \\
\hline \multirow[t]{2}{*}{ IN02 } & 256 & 1463 & NZ_CP035320.1 & $n f_{S A}$ & 693InsIS1R & Interruption & NA \\
\hline & & & (BR02) & $n f_{s} B$ & $575 \mathrm{G}>\mathrm{A}$ & G192D & $-6.954 *$ \\
\hline \multirow[t]{2}{*}{ IN03 } & 128 & 73 & NZ_CP009072.1 & $n f_{s} A$ & $634 \mathrm{~T}>\mathrm{C}$ & W212R & $-12.647 *$ \\
\hline & & & (ATCC25922) & $n f_{s} B$ & 327InsIS10R-like & Interruption & NA \\
\hline \multirow[t]{2}{*}{ IN04 } & $\geq 512$ & 69 & NC_011751.1 & $n f_{s} A$ & $302 \mathrm{~T}>\mathrm{G}$ & L101R & $-3.030 *$ \\
\hline & & & (UMN026) & $n f_{s} B$ & 476:479delTGGA & L159fs & NA \\
\hline \multirow[t]{2}{*}{ IN05 } & 256 & 162 & NZ_CP035320.1 & $n f s A$ & 666delA & E223fs & NA \\
\hline & & & (BR02) & $n f_{s} B$ & $82 \mathrm{G}>\mathrm{T}$ & E28Stop & NA \\
\hline \multirow[t]{2}{*}{ IN06 } & 256 & 4219 & NZ_HG941718.1 & $n f s A$ & $635 \mathrm{G}>\mathrm{A}$ & W212Stop & NA \\
\hline & & & (EC958) & $n f_{s} B$ & $281 \mathrm{G}>\mathrm{A}$ & W94Stop & NA \\
\hline \multirow[t]{3}{*}{ IN07 } & $\geq 512$ & 919 & NC_007946.1 & $n f s A$ & $635 \mathrm{G}>\mathrm{A}$ & W212Stop & NA \\
\hline & & & (UTI89) & $n f s B$ & $137 \mathrm{G}>\mathrm{A}$ & W46Stop & NA \\
\hline & & & & $r i b E$ & $151 \mathrm{~A}>\mathrm{G}$ & $\mathrm{I} 51 \mathrm{~V}$ & 0.363 \\
\hline \multirow[t]{6}{*}{ IN08 } & $\geq 512$ & 685 & NZ_CP007265.1 & $n f s A$ & $121 \mathrm{G}>\mathrm{A}$ & G41S & 3.174 \\
\hline & & & (ST540) & & 123delT & L43fs & NA \\
\hline & & & & & $343 \mathrm{~T}>\mathrm{G}$ & S115A & 2.175 \\
\hline & & & & & $350 \mathrm{C}>\mathrm{T}$ & T117I & 0.634 \\
\hline & & & & $n f s B$ & $381 \mathrm{G}>\mathrm{A}$ & M127I & -0.888 \\
\hline & & & & & $458 \mathrm{G}>\mathrm{A}$ & G153D & $-6.995 *$ \\
\hline \multirow[t]{2}{*}{ IN09 } & 256 & 73 & NZ_CP009072.1 & $n f_{s} A$ & $199 \mathrm{C}>\mathrm{T}$ & Q67Stop & NA \\
\hline & & & (ATCC25922) & $n f_{s} B$ & $113 \mathrm{C}>\mathrm{T}$ & P38L & $-8.693 *$ \\
\hline
\end{tabular}

149 MICs were determined using Etests (26). Isolate names of reference genomes are noted by parentheses

150 below the RefSeq accessions. Asterisks following PROVEAN scores denote deleterious mutations

151 determined when scores $\leq-2.5$. Notations: ST, (multi-locus) sequence type; NA, not applicable; ins, 152 insertion; del, deletion; fs, frameshift.

153 Interruption of $n f s A$ or $n f_{s} B$ by insertion sequences was identified in three genomes, IN01-03

154 (Table 1), using SPAdes (27) assembly graphs (Dataset S1) and was then confirmed using Sanger 
155 sequencing of PCR products (Fig. 2). Annotations and interrupted genetic regions of these insertion 156 sequences are available at github.com/wanyuac/NITREc/tree/master/Seq/Nucl/IS. Specifically, $n f s A$ 157 in both IN01 and IN02 was interrupted by ISIR (768 bp, IS 1 family) encoded on the reverse 158 complementary strand. No nucleotide divergence was seen between these two copies of IS $1 R$ and their 159 reference sequence (GenBank accession: AH003427.2; region: 3,877-4,644). Nine-bp direct repeats 160 (DRs) flanking ISIR revealed its insertion site between bases 693 and 694 of $n f s A$. Similarly, $n f s B$ in 161 IN03 was interrupted by an IS10R-like element (1,329 bp, IS4-family) that differed from IS10R 162 (GenBank accession: AH003348.2; bases 867-2,195) by 12 nucleotides, consisting of two nucleotide 163 substitutions in the right inverted repeat (IRR) and 10 missense mutations that resulted in four amino 164 acid substitutions in the transposase gene. This element was inserted between bases 327 and 328 of $165 n f s B$, as indicated by its flanking 9-bp DRs, and in an opposite orientation to $n f s B$. A search of this 166 insertion sequence against GenBank (accessed in August 2020) found two exact matches in E. coli 167 genomes of animal and clinical origins, respectively (accessions: CP009578.1 and CU928145.2). A 168 complete Tn10 variant (GenBank accession: AF162223.1) (28), bounded by an IS 10L-like element 169 and IS10R, was also identified in the assembly graph of IN03. Notably, this ISIOL-like element only 170 differed from the IS10R-like element of IN03 by four nucleotide substitutions in their left inverted 171 repeats (IRLs). Assembly depths and connections of nodes consisting of the IS $10 L$-like element, IS10R, 172 and Tn10 variant in the assembly graph of IN03 genome suggested high copy numbers of both insertion 173 sequences and only a single copy of Tn10. Taken together, although the transposon did not integrate 174 into $n f s B$, replicative transposition of its IS $10 L$ or IS $10 R$ component may cause the interruption of $n f s B$. 


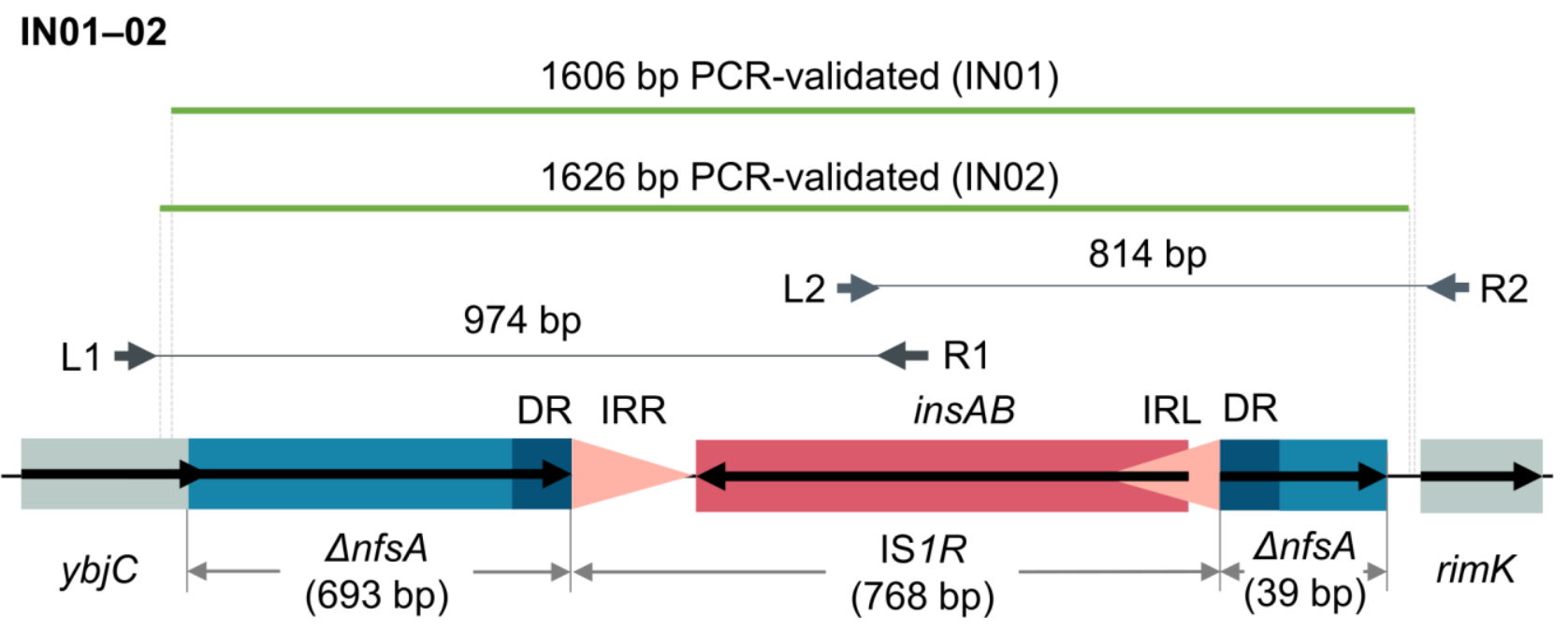

IN03

2205 bp PCR-validated

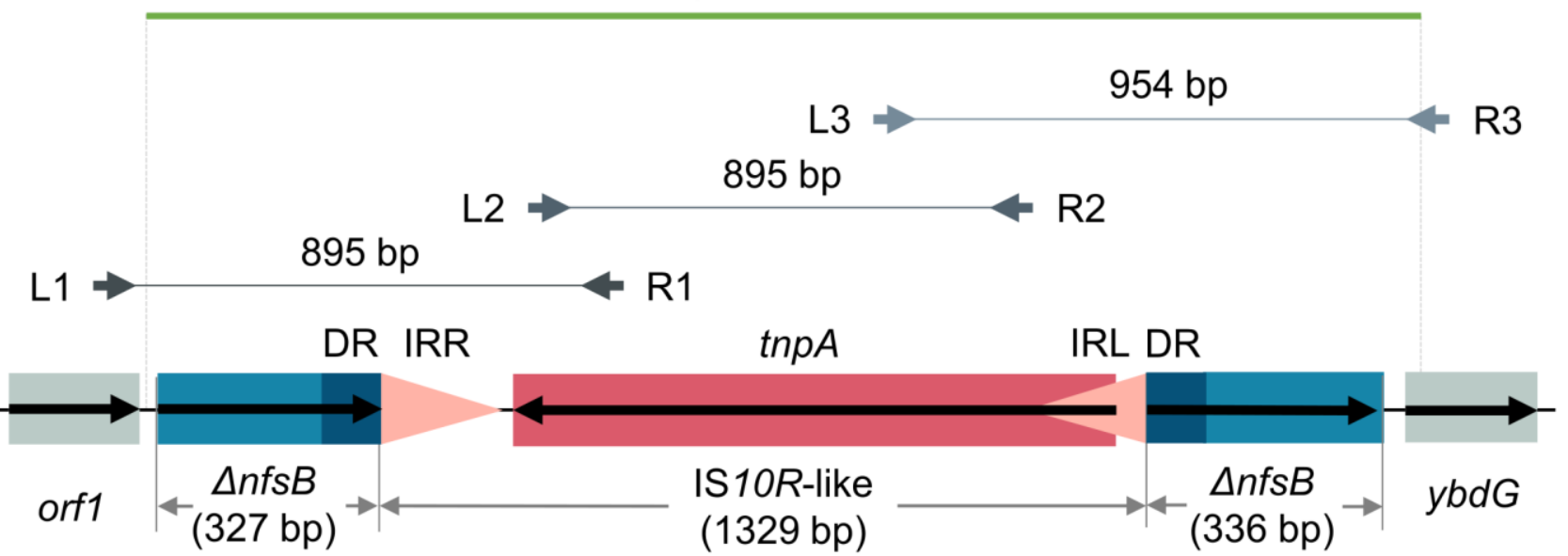

176 FIG 2 Interruption of $n f_{s} A$ and $n f_{s} B$ by insertion sequences. Orientation of each coding sequence is

177 indicated by a black bold arrow. IRL and IRR (inverted repeat left and right) of each insertion sequence

178 are represented by pink triangles indicating their orientations. Direct repeats (DRs) flanking each

179 insertion sequence are denoted by dark-blue boxes. These genetic structures were confirmed by Sanger

180 sequencing of PCR products. Paired left and right PCR primers are denoted by grey bold arrows above

181 DNA backbones and are aligned to their source positions. Each pair of primers are connected by a grey

182 solid line to indicate their expected PCR product. Consensus sequences of Sanger reads are denoted

183 by green solid lines. Note that consensus sequences of IN01 and IN02 differed in lengths due to quality

184 trimming of the reads. 
186 A single copy of ribE in each of IN01-09 genomes was predicted from read mapping. Genome IN07 187 carried the only amino acid substitution identified in RibE (I51V, compared to the reference sequence 188 of genome UTI89), which was attributed to a missense mutation 151A>G (Table S2). Nonetheless, 189 this RibE variant was identical to RibE sequences from another five nitrofurantoin-susceptible 190 reference genomes (ATCC25922, BR02, EC958, ST540, and UMN026). Therefore, the effect of 191 substitution I51V was considered neutral, further supported by PROVEAN prediction (score: 0.363 ).

\section{Horizontally acquired nitrofurantoin resistance}

193 No evidence of horizontally acquired nitrofurantoin resistance was identified in isolates IN01-09 as 194 neither the known genetic determinant $o q x A$ nor $o q x B$ was identified in any of their genomes. 195 Nonetheless, other acquired AMR genes were present in genomes of IN01-05 but not IN06-09 (Tables 1962 and S2), conferring resistance to aminoglycosides, beta-lactams, trimethoprim, sulphonamides, and tetracyclines. With one exception ( $d f r A 1$ in IN04), alleles of these genes were identical to the respective reference sequences.

199 TABLE 2 Alleles of acquired AMR genes detected in genomes of isolates IN01-05.

\begin{tabular}{|c|c|c|c|c|c|c|c|c|c|c|}
\hline Isolate & $\operatorname{aadA1}$ & dfrA1 & sul1 & $\operatorname{aph}\left(3^{\prime}\right)-I a$ & $\operatorname{aph}\left(3^{\prime \prime}\right)-I b$ & $\operatorname{aph}(6)-I d$ & sul2 & $\operatorname{tet}(\mathbf{A})$ & $\operatorname{tet}(\mathbf{B})$ & bla TEM \\
\hline IN01 & $\operatorname{aadAl}$ & $d f r A l$ & sull & $\operatorname{aph}\left(3^{\prime}\right)-I a$ & $a p h\left(3^{\prime \prime}\right)-I b$ & $\operatorname{aph}(6)-I d$ & sul2 & $\operatorname{tet}(\mathrm{A})$ & $\operatorname{tet}(\mathrm{B})$ & - \\
\hline IN02 & $\operatorname{aadAl}$ & $d f r A l$ & sull & $\operatorname{aph}\left(3^{\prime}\right)-I a$ & $a p h\left(3^{\prime \prime}\right)-I b$ & $\operatorname{aph}(6)-I d$ & sul2 & $\operatorname{tet}(\mathrm{A})$ & $\operatorname{tet}(\mathrm{B})$ & - \\
\hline IN03 & - & - & - & - & - & - & - & - & $\operatorname{tet}(\mathrm{B})$ & - \\
\hline IN04 & $\operatorname{aadAl}$ & $d f r A l^{*}$ & sull & - & - & - & - & - & - & $b l a_{\mathrm{TEM}-1 \mathrm{~B}}$ \\
\hline IN05 & - & - & - & - & - & - & - & - & - & $b l a_{\mathrm{TEM}-1 \mathrm{~B}}$ \\
\hline
\end{tabular}

200 Allele labels in column names denote clusters of reference sequences based on a minimum nucleotide 201 identity of $80 \%$. Allele $d f r A l *$ differed from its best-matched reference allele ( $\left.d f r A 1.9 \_A J 238350\right)$ in the ResFinder database (29) by one nucleotide change causing an amino acid substitution P65S. 
203 Diversity of nitrofurantoin-resistance determinants in UK E. coli

204 To contextualise genetic alterations identified in the nine genomes IN01-09, genomes of additional 12,403 UK E. coli isolates were retrieved, consisting of 2,009 blood, urine, and gut microbiota isolates from an NIHR Health Protection Research Unit (HPRU) collection; 1,509 blood isolates collected by the British Society for Antimicrobial Chemotherapy (BSAC) or the Cambridge University Hospitals NHS Foundation Trust (hereafter, BSAC or CUH collection) (30); 297 isolates from East England project (33), including historic isolates; and 8,341 isolates recorded in EnteroBase (34) (Table S3). 12,410 genomes; 12,393 hits for $n f s B$ in 12,388 genomes; and 12,417 hits for ribE in all 12,412 genomes. Notably, $n f s A$ was not detected in two genomes, and $n f s B$ was not detected in another 24 genomes. By contrast, 75 genomes (including IN01-02) had 2-4 hits for $n f s A$ (complete or partial coding sequence); five genomes (including IN03) had two hits for $n f_{s} B$ (partial); and five genomes had two hits for ribE (complete). However, none of the 12,412 genomes had more than one hit for any two or all of the genes $n f s A, n f s B$, and $r i b E$.

A decision tree was developed to categorise hits for each gene based on predicted protein sequences identified, which failed to encode complete 240-aa (amino acid) NfsA proteins. Frameshift mutations were present in all 107 alleles with indels, resulting in predicted proteins of 18-247 aa. Nonsense mutations identified in 117 alleles encoded partial NfsA proteins of 23-234 aa. Interestingly, amongst missense mutations, the start codon ATG of $n f s A$ was altered in 14 genomes: mutation M1T $(2 \mathrm{~T}>\mathrm{C})$ was found in an allele shared by two BSAC isolates eo393 and eo2899 (collected in 2002 and 2010, respectively), causing a loss of start codon; start-codon substitution M1I (3G>A) was found in five $n f s A$ alleles from 12 genomes (Table S4) and was considered deleterious to protein structure according to the PROVEAN prediction (uniform score: -3.149 ). 

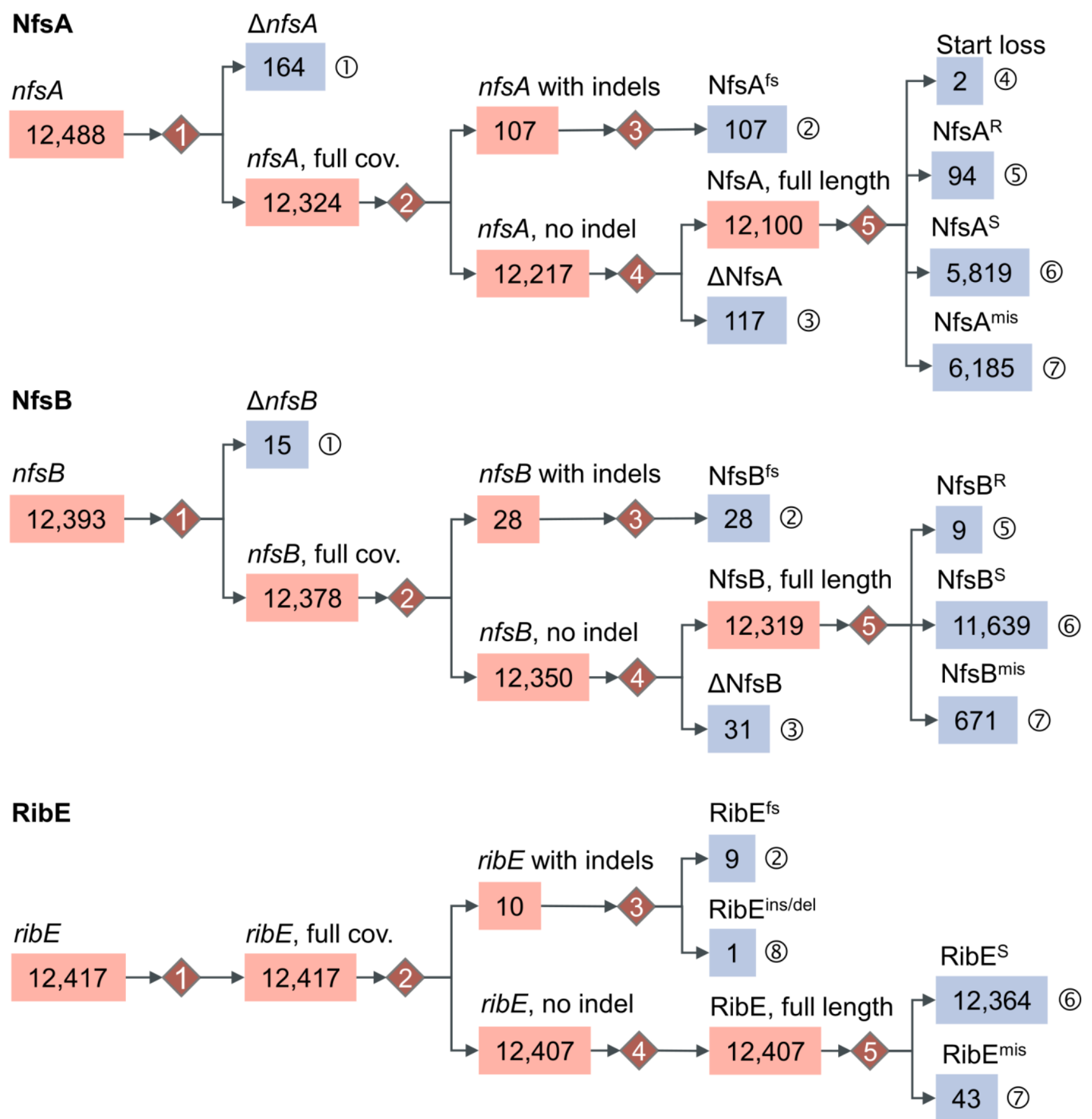

FIG 3 Classification of $n f_{s} A, n f s B$, and ribE alleles from 12,412 E. coli genomes, based on nucleotide-

230 and protein-level comparisons. Final allele categories are labelled with (1)-(8) and shaded in blue. These

231 categories are determined at five decision points indicated by numbered red diamonds. Numbers of 232 alleles in each category are displayed in boxes. Categories with zero counts are omitted for 233 convenience. Assessment at each decision point: (1) Does the allele sequence cover $100 \%$ of its 234 reference sequence? (2) Is there any gap opened in the sequence alignment? (3) Is there a frameshift 235 mutation or insertion/deletion of any codon? (4) Is there any nonsense mutation? (5) Does the predicted 
protein sequence carry no mutation, or any known resistance-associated amino acid substitution, or mutations known to be associated with nitrofurantoin resistance; $\mathrm{S}$, proteins identical to those in

240 nitrofurantoin susceptible isolates; mis, missense mutations of unknown phenotypical impacts.

Genetic alterations of $n f s B$ (654 bp) displayed a similar pattern to $n f s A$. Particularly, 15 partial sequences (35-647 bp) were identified and no start-codon loss was seen amongst missense mutations

243 (Table S4). By contrast, ribE (471 bp) displayed lower diversity than the other two genes, with no 244 partial allele sequence or nonsense mutation identified. At least one copy of ribE was detected in each genome.

Among ribE alleles identified in the 12,412 genomes, a stop-codon substitution (TGA>TAA) 248 Both alleles encoded the same RibE protein as the nitrofurantoin-susceptible strain ATCC25922 249 (NCBI protein accession: WP_001021161.1). A novel deletion of four amino acid residues (KAGN, 250 from position 132 to 135 in the reference RibE sequence from the ATCC25922 genome) was predicted 251 from one (carried by isolate EC0430U) of $10 \mathrm{ribE}$ alleles that possessed indels. Notably, this deletion 252 overlapped the deletion of amino acid residues 131-134 (TKAG, from the same reference RibE 253 sequence) that is known to reduce nitrofurantoin susceptibility (7). Putatively deleterious missense 254 mutations of RibE were identified in three genomes of further HPRU isolates: EC0340B (A16V), EC0444B (A34T), and EC1165B (T131S), with PROVEAN scores of -3.406, -3.893, and -2.742, 256 respectively (Table S4).

Acquired resistance genes $\operatorname{oq} x A(1,176 \mathrm{bp})$ and $\operatorname{oq} x B(3,153 \mathrm{bp})$ were rare among the 12,412 258 genomes: both genes were detected in only one genome (eo1692, from the BSAC collection), with 
exact matches to their reference sequences in the ResFinder database (29). No other hit for either gene was obtained given minimum nucleotide coverage and query coverage of $70 \%$ and $80 \%$, respectively.

\section{Evolution of $\boldsymbol{n f s} \boldsymbol{A}, \boldsymbol{n} \boldsymbol{f} \boldsymbol{s} \boldsymbol{B}$, and $\boldsymbol{r i b E}$}

262 Based on the observed diversity of these three genes in the 12,412 E. coli genomes, we hypothesised 263 that $r i b E$ was under stronger negative or purifying selection than $n f s A$ and $n f s B$. The estimated ratio 264 for the nucleotide nonsynonymous-substitution rate $(\mathrm{dN})$ over synonymous-substitution rate (dS), 265 denoted as $\omega=d N / d S$, was $0.607812,0.40739$, and 0.179068 for $n f s A, n f s B$, and ribE, respectively, 266 indicating that all three genes were under purifying selection with ascending strength in the order $n f s A$ $<n f s B<r i b E$.

After deduplicating identical alleles from the 12,412 genomes, 231 (32\% of $723 \mathrm{bp}$ ), 184 (28\% of $654 \mathrm{bp}$ ), and $86(18 \%$ of $471 \mathrm{bp})$ SNP sites were found in $328 n f s A$ alleles, $231 n f s B$ alleles, and 88 ribE alleles, respectively (Figures S1-3). Despite this variation, phylogenetic trees of these three genes generally showed extremely low bootstrap values (for instance, $0-2$ ) in the majority of ancestral reliably reconstructing the evolutionary history of each gene.

Pairwise homoplasy indexes (PHIs) calculated from the allele alignment of each gene revealed significant homoplasy in $n f s A$ (p-value: 0.0434 ) and $n f s B$ (p-value: 0.0363 ) but not in ribE (p-value:

0.736). Comparisons of each allele alignment to its corresponding gene tree identified 82, 50 , and 12

\section{Prediction of nitrofurantoin susceptibility and experimental validation}

279 For each of the 2,009 HPRU isolates, which we had access to, nitrofurantoin susceptibility was 280 predicted from sequence categories (Fig. 3) of intrinsic genes $n f s A, n f s B$, ribE and presence-absence 281 of both acquired genes $o q x A$ and $o q x B$ using a scoring algorithm (Section "Materials and Methods"). 282 Specifically, the algorithm gave a risk score $r(0 \leq r \leq 4)$ to each isolate as a summary of its five-gene 
$283(n f s A, n f s B$, ribE, oqxA, and $o q x B)$ sequence categories or genotypes. We hypothesised that a higher

284 score would predict a greater reduction in the nitrofurantoin susceptibility of an isolate, and vice versa.

285 Of note, neither $o q x A$ nor functional $o q x B$ was detected in any of these isolates.

286 Using $r \geq 1$ as a criterion, a non-redundant set of 62 HPRU E. coli isolates (one isolate per patient)

287 were predicted to have reduced nitrofurantoin susceptibility (Table S5). Since RibE sequences of these

288 isolates were identical to those of wildtype RibE proteins in nitrofurantoin-susceptible isolates, 16

289 genotypes associated with reduced nitrofurantoin susceptibility were identified in these 62 isolates

290 based on $n f_{S A}$ and $n f s B$ sequences only (Table 3).

291 TABLE $3 n f s A-n f s B$ genotypes of $E$. coli isolates with nitrofurantoin-resistance risk scores $\geq 1$.

\begin{tabular}{|c|c|c|c|c|c|c|c|}
\hline & \multicolumn{7}{|c|}{ Isolate number of each genotype (number selected for experimental validation) } \\
\hline${ }_{n f s A} n f s B$ & Wildtype & $\begin{array}{l}\text { Missense, } \\
\text { known }\end{array}$ & $\begin{array}{l}\text { Missense, } \\
\text { unknown }\end{array}$ & Nonsense & Frameshift & Fragmented & Absent \\
\hline Wildtype & 0 & $1(1)$ & 0 & 0 & $1(1)$ & 0 & 0 \\
\hline $\begin{array}{l}\text { Missense, } \\
\text { known }\end{array}$ & $9(3)$ & 0 & $3(2)$ & $1(1)$ & 0 & 0 & $1(1)$ \\
\hline $\begin{array}{l}\text { Missense, } \\
\text { unknown }\end{array}$ & 0 & 0 & 0 & 0 & $2(1)$ & 0 & $1(1)$ \\
\hline Nonsense & $13(1)$ & $1(1)$ & $1(1)$ & 0 & $1(1)$ & 0 & 0 \\
\hline Frameshift & $13(1)$ & 0 & 0 & 0 & 0 & 0 & 0 \\
\hline Fragmented & $11(1)$ & $1(1)$ & $2(2)$ & 0 & 0 & 0 & 0 \\
\hline
\end{tabular}

292 Genotypes of 62 non-redundant HPRU E. coli isolates are shown. A gene was considered fragmented

293 when it was interrupted or truncated. In total, 16 genotypes had at least one isolate each, and 20 isolates

294 were selected for experimental validation.

Assuming nonsense or frameshift mutations observed in this study always cause a loss of function, twenty representative isolates of the 16 genotypes were selected from the 62 isolates as a "prediction 
298 was chosen for each genotype that did not involve any missense mutation. Otherwise, one

299 representative was chosen for each missense mutation. In this group, all 12 double mutants carried at

300 least one resistance-associated genetic alteration $(1<r \leq 2)$. Nitrofurantoin susceptibility was correctly

301 predicted for all these isolates. Notably, the double mutants were highly resistant to nitrofurantoin

302 (MIC $\geq 256 \mathrm{mg} / \mathrm{L}$ ) when both genetic alterations were known to be associated with nitrofurantoin

303 resistance. Predictions for the eight single mutants of resistance-associated genetic alterations $(r=1)$

304 were less reliable than those for double mutants, with five single mutants being more susceptible to

305 nitrofurantoin than predicted. Isolates EC394_9, EC0880B, and EC0026B showed heterogenous

306 responses to nitrofurantoin as a few colonies of resistant E. coli appeared within inhibition zones.

307 TABLE 4 Genotypes and confirmed nitrofurantoin susceptibility of E. coli isolates in test groups.

\begin{tabular}{|c|c|c|c|c|c|c|c|c|}
\hline \multirow{2}{*}{ Group } & \multirow{2}{*}{ Isolate } & \multirow{2}{*}{$\begin{array}{l}\text { NfsA } \\
(240 \text { aa) }\end{array}$} & \multirow{2}{*}{$\begin{array}{l}\text { NfsB } \\
(217 \text { aa })\end{array}$} & \multirow{2}{*}{$\begin{array}{l}\text { RibE } \\
(156 \text { aa) }\end{array}$} & \multirow{2}{*}{$\boldsymbol{r}$} & \multicolumn{2}{|c|}{ Susceptibility } & \multirow{2}{*}{$\begin{array}{l}\text { MIC } \\
(\mathrm{mg} / \mathrm{L})\end{array}$} \\
\hline & & & & & & Predicted & Observed & \\
\hline \multirow{20}{*}{$\begin{array}{l}\text { Prediction } \\
\text { (20 isolates) }\end{array}$} & EC0064B & G154E & Wildtype & Wildtype & 1.0 & $\mathrm{~S}(\mathrm{I})$ & $\mathrm{S}(\mathrm{I})$ & 64 \\
\hline & EC0890B & H11Y & Wildtype & Wildtype & 1.0 & $\mathrm{~S}(\mathrm{I})$ & $\mathrm{S}$ & 16 \\
\hline & EC1069B_1 & G126R & Wildtype & Wildtype & 1.0 & $\mathrm{~S}(\mathrm{I})$ & $\mathrm{S}$ & 32 \\
\hline & EC394_9 & Q67Stop & Wildtype & Wildtype & 1.0 & $\mathrm{~S}(\mathrm{I})$ & $\mathrm{S}(\mathrm{I})$ & $64 *$ \\
\hline & EC0880B & 1:421 int413:723 & Wildtype & Wildtype & 1.0 & $\mathrm{~S}(\mathrm{I})$ & $\mathrm{R}$ & $256 *$ \\
\hline & EC1161B & I228fs & Wildtype & Wildtype & 1.0 & $\mathrm{~S}(\mathrm{I})$ & $\mathrm{S}$ & 32 \\
\hline & EC0932B & Wildtype & F84S & Wildtype & 1.0 & $\mathrm{~S}(\mathrm{I})$ & $\mathrm{S}$ & 4 \\
\hline & EC1187B & Wildtype & Y183fs & Wildtype & 1.0 & $\mathrm{~S}(\mathrm{I})$ & $\mathrm{S}$ & 32 \\
\hline & $E C 0026 B$ & Q113Stop & H80Y & Wildtype & 1.1 & $\mathrm{~S}(\mathrm{I})$ or $\mathrm{R}$ & $\mathrm{S}(\mathrm{I})$ & $64 *$ \\
\hline & $E C 0067 B$ & 1:308int $434: 723$ & W46R & Wildtype & 1.1 & $\mathrm{~S}(\mathrm{I})$ or $\mathrm{R}$ & $\mathrm{R}$ & $>512$ \\
\hline & $E C 0179 B$ & G126R & W94Stop & Wildtype & 2.0 & $\mathrm{R}$ & $\mathrm{R}$ & $>512$ \\
\hline & $E C 0328 B$ & G126R & Absent & Wildtype & 2.0 & $\mathrm{R}$ & $\mathrm{R}$ & 256 \\
\hline & $E C 0363 B$ & R133C & T88fs & Wildtype & 1.1 & $\mathrm{~S}(\mathrm{I})$ or $\mathrm{R}$ & $\mathrm{R}$ & 128 \\
\hline & $E C 0439 B$ & H11Y & W46R & Wildtype & 1.1 & $\mathrm{~S}(\mathrm{I})$ or $\mathrm{R}$ & $\mathrm{R}$ & $>512$ \\
\hline & EC0553Bo & $\mathrm{S} 38 \mathrm{~F}$ & Absent & Wildtype & 1.1 & $\mathrm{~S}(\mathrm{I})$ or $\mathrm{R}$ & $\mathrm{R}$ & $>512$ \\
\hline & EC0629B & $1: 52 \mathrm{del}$ & G192S & Wildtype & 2.0 & $\mathrm{R}$ & $\mathrm{R}$ & 512 \\
\hline & $E C 0644 B$ & 1:69del & P209L & Wildtype & 1.1 & $\mathrm{~S}(\mathrm{I})$ or $\mathrm{R}$ & $\mathrm{S}(\mathrm{I})$ & 64 \\
\hline & $E C 0812 U$ & W159Stop & L22fs & Wildtype & 2.0 & $\mathrm{R}$ & $\mathrm{R}$ & $>512$ \\
\hline & $E C 0856 B$ & G131D & K205E & Wildtype & 1.1 & $\mathrm{~S}(\mathrm{I})$ or $\mathrm{R}$ & $\mathrm{R}$ & $>512$ \\
\hline & $E C 1131 B$ & E75Stop & G192D & Wildtype & 2.0 & $\mathrm{R}$ & $\mathrm{R}$ & $>512$ \\
\hline \multirow{5}{*}{$\begin{array}{l}\text { Exploration } \\
\text { (7 isolates) }\end{array}$} & EC6002_8 & G41S & Wildtype & Wildtype & 0.1 & $\mathrm{~S}$ or $\mathrm{S}(\mathrm{I})$ & $\mathrm{S}$ & 8 \\
\hline & $E C 1146 B$ & G66R & Wildtype & Alt. stop & 0.1 & $\mathrm{~S}$ or $\mathrm{S}(\mathrm{I})$ & $\mathrm{S}$ & 32 \\
\hline & EC6125_8 & Wildtype & M127I & Wildtype & 0.1 & $\mathrm{~S}$ or $\mathrm{S}(\mathrm{I})$ & $\mathrm{S}$ & 16 \\
\hline & EC0340B & Wildtype & Wildtype & A16V & 0.1 & $S$ or $S(I)$ & $\mathrm{S}$ & 16 \\
\hline & EC0430U & Wildtype & Wildtype & 132:135del & 0.1 & $\mathrm{~S}$ or $\mathrm{S}(\mathrm{I})$ & $\mathrm{S}(\mathrm{I})$ & 64 \\
\hline
\end{tabular}




\begin{tabular}{lllllllll} 
& EC0444B & Wildtype & Wildtype & A34T & 0.1 & S or S(I) & S & 32 \\
& EC1165B & Wildtype & Wildtype & T131S & 0.1 & S or S(I) & S & 8 \\
Control (-) & ATCC25922 & Wildtype & Wildtype & Wildtype & 0.0 & S & S & 16 \\
Control (+) & IN09 & Q67Stop & P38L & Wildtype & 1.1 & S(I) or R & R & 256 \\
\hline
\end{tabular}

308 Lengths of wildtype NfsA, NfsB, and RibE are noted beneath protein names. Names of isolates

309 carrying two mutations (namely, double mutants) are italicised. Known genetic alterations associated

310 with nitrofurantoin resistance (Tables 1, A1, and A2) are shaded in red. Genetic alterations identified

311 in IN01-09 genomes are boldfaced. Asterisks indicate isolates of which colonies within inhibition

312 zones were seen and considered when determining the MICs. Interpretation of MICs: S, susceptible

$313(\mathrm{MIC} \leq 32 \mathrm{mg} / \mathrm{L}) ; \mathrm{S}(\mathrm{I})$, susceptible, under increased exposure to nitrofurantoin $(32 \mathrm{mg} / \mathrm{L}<\mathrm{MIC} \leq 64$

$314 \mathrm{mg} / \mathrm{L}) ; \mathrm{R}$, resistant (MIC > $64 \mathrm{mg} / \mathrm{L})$. Nitrofurantoin susceptibility of the negative (-) and positive (+)

315 controls must not be used as support for validation because their $n f s A, n f s B$, and $\operatorname{ribE}$ alleles were used

316 as references by the prediction algorithm. Abbreviations: int, gene interruption, with nucleotide

317 positions (start : end) of remnants related to their wildtype references noted aside; del, deletion or

318 truncation, with its start and end nucleotide positions noted aside; alt. stop, alternative stop codon.

319 Seven additional isolates were included as an exploratory group to examine potential effects of 320 novel mutations on nitrofurantoin susceptibility (Table 4): NfsA or NfsB of two isolates had a missense 321 mutation that had also been identified in isolate IN08; ribE of one isolate was terminated by an 322 alternative stop codon TAA; and another four isolates had mutations in RibE, while genomes of all the 323 four isolates encoded wildtype $\mathrm{NfsA}$ and $\mathrm{NfsB}$. A nitrofurantoin-resistance risk score of 0.1 was 324 assigned to each isolate by the prediction algorithm to denote presence of a novel mutation. All seven 325 isolates were susceptible to nitrofurantoin (MICs $\leq 64 \mathrm{mg} / \mathrm{L}$ ), matching our predictions. None of the 326 six missense mutations resulted in nitrofurantoin resistance. By contrast, isolate EC0430U, which 327 carried a deletion of $\mathrm{KAGN}^{132-135}$ in RibE, showed an MIC of $64 \mathrm{mg} / \mathrm{L}$, exceeding that of a previously 328 reported isolate having an overlapping 4-aa deletion in RibE (7). 


\section{Predicted occurrences of reduced nitrofurantoin susceptibility in UK E. coli blood isolates}

330 As data relating to nitrofurantoin susceptibility among bacteriaemia E. coli isolates is seldom available,

331 occurrences of reduced nitrofurantoin susceptibility were calculated for a non-redundant collection of 332 2,253 bacteriaemia E. coli isolates that were collected in 2001-2016 from across the UK, predating 333 the change in national guidelines. This collection comprised 582 HPRU isolates (one isolate was 334 selected per ST per patient), 1,509 BSAC or CUH isolates, and 162 SCOT isolates. Overall, $142(6.3 \%)$ 335 isolates were predicted to show reduced nitrofurantoin susceptibility. Specifically, 123 (5.5\%) single336 mutation isolates had a resistance-associated alteration in either $n f s A$ (117 isolates, 5.2\%) or $n f s B(6$ 337 isolates, $0.3 \%)$ and $19(0.8 \%)$ double-mutation isolates had resistance-associated alterations in both $338 n f s A$ and $n f s B$.

\section{Discussion}

340 In this study, we have explored genetic determinants of nitrofurantoin resistance in UK E. coli starting 341 with nine sequenced nitrofurantoin-resistant clinical isolates and a comparative genomics approach. 342 We found four types of alterations in two intrinsic, oxygen-insensitive nitroreductase genes ( $n f s A$ and $343 n f s B$ ) that are known to be associated with nitrofurantoin resistance: gene interruptions by insertion 344 sequences, frameshift mutations caused by indels, nonsense mutations and missense mutations both 345 caused by single-nucleotide substitutions. Notably, each of these nine isolates had alterations in both $346 n f s A$ and $n f s B$, equivalent to double-step or multi-step mutants of both genes, and hence explaining 347 high nitrofurantoin MICs ( $\geq 128 \mathrm{mg} / \mathrm{L})$ of these isolates (35).

348 Both $n f s A$ and $n f s B$ had at most one missense mutation identified in genomes of isolates IN01-07 349 and IN09, and these mutations were predicted to be deleterious to protein functions by PROVEAN 350 (Table 1). Mutation W212R of NfsA in isolate IN03 was previously identified in an Iranian 351 nitrofurantoin-resistant $E$. coli isolate EC168 (MIC $\geq 512 \mathrm{mg} / \mathrm{L}$ ), in which no functional $n f s B$ gene was 352 detected (36). Since nucleotide substitutions differ between IN03 (634T>C) and EC168 (634T>A), 
353 this mutation may have arisen independently in each isolate. $\mathrm{W}^{212}$, located in $\alpha$-helix $\alpha 10$, forms the

354 active site of NfsA (Protein Data Bank or PDB accession for protein structure: 1F5V) with the

355 hydrophobic side chain of tryptophan and is conserved when compared with the NfsA counterparts in

356 Vibrio harveyi and Bacillus subtilus (37, 38). A functional disruption can hence be anticipated when

357 substituting this side chain with the positively charged side chain of arginine. The other missense

358 mutation of NfsA identified in our study, L101R in isolate IN04, is novel and, as in the case of EC168,

359 this mutation possibly disrupts the function of NfsA because IN04 was highly resistant to

360 nitrofurantoin (MIC $\geq 512 \mathrm{mg} / \mathrm{L}$ ) and did not possess a functional $n f s B$ gene. Since $\mathrm{L}^{101}$ is part of $\alpha$ -

361 helix $\alpha 6$ in the central domain of NfsA $(37,38)$, substitution of this hydrophobic, non-polar residue

362 with arginine may have a disruptive impact on the protein structure and function.

Mutation G192D in NfsB from isolates IN01 and IN02 was previously identified in a

nitrofurantoin-resistant E. coli isolate (collected in 1999-2000) having a deletion in $n f s A$ and showing

a nitrofurantoin MIC of $128 \mathrm{mg} / \mathrm{L}(35,39)$; substitution of $\mathrm{G}^{192}$ with alanine has also been associated

with nitrofurantoin resistance (35). Located at the end of the fourth $\beta$-sheet of NfsB (PDB accession:

1DS7), $\mathrm{G}^{192}$ constitutes the hydrophobic core that accommodates co-factor flavin mononucleotide

$(\mathrm{FMN})(40)$ and is in close spatial proximity of negatively charged residue $\mathrm{D}^{160}$. Therefore, substitution

369 of this neutral, hydrophobic residue with another aspartic acid residue possibly affects the protein

370 formation as well as its function. A similar impact of the NfsB mutation G153D in isolate IN08 on the

371 protein function is anticipated based on the same difference in hydrophobicity of amino acid residues.

372 Nonsense mutations, frameshift mutations, and insertion sequence mediated interruptions of $n f s A$

373 or $n f s B$ are also common loss-of-function genetic alterations causing nitrofurantoin resistance $(35,36$,

374 41) and we found examples of all these alterations in isolates IN01-09 (Table 1). The interruption of

$375 n f_{S A}$ by an IS 1 -family insertion sequence has been reported by three other studies, two of which also

376 identified insertion of IS 1 in $n f s B$, while one identified integration of the composite transposon $\operatorname{Tn} 10$

377 into $n f s A(6,7,35)$. Interestingly, the position and flanking nucleotides of the IS1 insertion site differ 
among all three studies, indicating variability of IS 1 in gene inactivation and being consistent with the known AT-rich specificity of IS 1 for target sites (42). In comparison, the interruption of $n f s B$ by a novel IS10R-like insertion sequence had not been reported before, although another IS4-family insertion sequence (IS 186) was found in disrupted $n f s A$ (6). Since IS 10-group elements comprise both ends of $\mathrm{Tn} 10$, the presence of Tn10 in isolate IN03 implies transposition of IS $10 R$ or its IS $10 L$-like element from $\mathrm{Tn} 10$ to $n f s A$ or $n f s B$, conferring reduced nitrofurantoin susceptibility to host bacteria.

384 Nevertheless, we anticipate that it is less likely for IS10L (left end of Tn10) to show the same behaviour as IS10R because its transposition function is much weaker than the latter (43). We might see a growing frequency of insertion sequence mediated nitrofurantoin resistance in the future, if copy numbers of

IS 1 - and IS4-family elements in E. coli genomes are undergoing similar accumulation trajectories as those in Shigella spp (44).

This study has revealed variation of genes $n f s A$, $n f s B$, and ribE among UK E. coli isolates. Identified alleles of these genes (Fig. 3) can be summarised into two primary types: functional alleles and pseudogenes. The latter can result from gene truncation, insertion sequence mediated gene interruption, frameshift mutations, nonsense mutations, as well as deleterious missense mutations, and confers reduced nitrofurantoin susceptibility to $E$. coli. Comparisons between $\mathrm{dN} / \mathrm{dS}$ ratios of $n f s A$, $n f s B$, and $r i b E$ support our hypothesis that $r i b E$ is subjected to the strongest pressure of negative selection whereas $n f s A$ is subjected to the weakest. Not only does this variation in selective pressures explain the observed diversity difference between these three genes in both this study and literature 398 increments of nitrofurantoin-resistance levels, which has been reported by several studies $(6,7,35)$. 399 Notably, $n f s A$ and $n f s B$ are classified as non-essential genes of $E$. coli, whereas ribE is considered as 400 an essential gene by the PEC database (shigen.nig.ac.jp/ecoli/pec) (45). The higher level of 401 evolutionary conservation of $r i b E$ compared with $n f s A$ and $n f s B$ appears to represent a greater degree 
402 of functional constraint on its sequence (46). Nonetheless, expression levels of these three genes might 403 also be important to consider (47).

404 Homoplasic SNPs were identified in $n f_{s} A, n f s B$, and ribE of E. coli isolates in our collection 405 (Figures S1-3), and recombination within $n f_{s} A$ and $n f s B$ was also inferred by the pairwise homoplasy 406 indexes. Both factors may contribute to the lack of congruent phylogenetic signals in alleles of each 407 gene (represented as low bootstrap values of branches in each gene tree), indicating that, in addition 408 to recombination, resistance mutations can arise independently in each gene across the E. coli 409 population. Given the observed extremely low occurrence of acquired resistance gene complex oqxAB 410 in our E. coli collection, de novo mutations of chromosomal genes $n f s A, n f s B$, and ribE; and insertion 411 sequence mediated interruptions of $n f s A$ and $n f s B$ may constitute the main source of reduced 412 nitrofurantoin susceptibility in UK E. coli isolates.

413 We developed a decision-tree based algorithm for predicting nitrofurantoin susceptibility from five 414 loci ( $n f s A, n f s B$, ribE, oxqA, and $o q x B$ ) that are known to be involved in nitrofurantoin resistance. 415 Nitrofurantoin-susceptibility testing showed that the algorithm correctly predicted susceptibility levels 416 for double mutants with known resistance-associated genetic alterations and for single mutants with 417 novel genetic alterations (Table 4). The discrepancy between predicted susceptibility and MICs of 418 isolates carrying single resistance-associated alterations (which include confirmed loss-of-function 419 alterations, such as nonsense and frameshift mutations, gene interruptions and truncations) in $n f s A$ or $420 n f s B$ suggests that impaired function or production of both oxygen-insensitive nitroreductases NfsA 421 and NfsB are required to render E. coli resistant to nitrofurantoin. This discrepancy also implies that 422 we may have overestimated the prevalence of nitrofurantoin resistance in the sequenced collection of 423 UK E. coli isolates, although the estimate of $6.3 \%$ is close to the observed prevalence of $5 \%$ in England 424 by $2019(10,16,17)$.

425 To provide reference information for future research, we have developed database NITREc 426 (github.com/wanyuac/NITREc), which will facilitate searching for the most closely related reference 
sequence of a query allele of $n f s A, n f s B$, or $\operatorname{ribE}$, minimising the number of reported variants and reducing noise in the results. We anticipate improvement in accuracy of both variant identification and susceptibility prediction when new references are incorporated into the database in the future.

This study has several limitations. First, the impact of novel missense mutations in $n f s A$ and $n f s B$ on nitroreductase function and bacterial fitness were not experimentally confirmed, for example,

432 through mutagenesis experiments. Such confirmation is desirable as the observed variation in MICs 433 of single mutants carrying missense mutations indicates that PROVEAN-predicted resistance 434 mutations may not cause reduced nitrofurantoin susceptibility and their effects may be offset by other 435 susceptibility mechanisms. Second, epidemiological analysis of nitrofurantoin-resistant E. coli is 436 limited owing to a lack of available information about nitrofurantoin susceptibility of E. coli isolates 437 in the UK or abroad. As such, several key clinical questions have not been addressed in this study, 438 including the association of STs or infection sites (e.g., blood, urinary tract, etc.) with nitrofurantoin 439 resistance, co- or cross-resistance between nitrofurantoin and other antimicrobials, and temporal trend 440 of nitrofurantoin resistance in the UK. Third, regulatory elements of the nitroreductase system or 441 DNA-repair mechanisms were not investigated in this study. Furthermore, untargeted analysis was not 442 carried out for identifying novel genetic mechanisms of nitrofurantoin resistance due to the small sample size of nitrofurantoin-resistant E. coli isolates that were available in this study.

\section{Conclusions}

445 We predict the major cause of nitrofurantoin resistance in UK E. coli to comprise sporadic de novo 446 mutations in chromosomal genes $n f s A, n f s B$, and $r i b E$; and interruptions of $n f_{s} A$ and $n f s B$ by insertion 447 sequences. Accordingly, clonal expansion of resistant mutants, mobilisation of insertion sequences, 448 and selection of resistant clones in the presence of nitrofurantoin are believed to be three driving forces 449 in the evolution of nitrofurantoin-resistant E. coli in the UK. Previous reports have suggested that $n f s A$ 450 and $n f_{s} B$ mutations are associated with significant fitness cost that may obstruct propagation of 
resistant $E$. coli $(35,48)$. However, it is notable that isolates investigated in these reports and the current

452 research were all identified from clinically significant infections, implying a fitness to survive in

453 enteric microbiota and to survive host immune response in the lower urinary tract. This fitness was

454 also supported by the possible community transmission identified with clonal isolates IN01 and IN02.

455 The same nitrofurantoin-resistance determinants of IN01-09 were also identified in bloodstream 456 isolates, underlining the ability of these E. coli to adapt to circumstance.

457 It is unclear whether nitrofurantoin resistance will become more prevalent as a result of increased 458 community nitrofurantoin exposure following the change in the national guideline. As such, routine 459 nitrofurantoin susceptibility testing and WGS of $E$. coli isolates are needed to monitor this trend. The 460 tools provided in our study will facilitate future WGS-based surveillance. Further work is required to 461 assess the fitness cost of resistance-associated genetic alterations and to identify novel mechanisms of 462 nitrofurantoin resistance.

\section{Materials and Methods}

\section{$464 \quad E$. coli isolates and antimicrobial susceptibility testing}

465 Automated EUCAST disc diffusion tests for nitrofurantoin susceptibility (Oxoid ${ }^{\mathrm{TM}}$ CT0034B 100- $\mu \mathrm{g}$ 466 nitrofurantoin discs, ThermoFisher ScientificTM, USA) in clinical urinary E. coli isolates were 467 routinely performed by a microbiology laboratory of the Imperial College Healthcare NHS Trust in 468 north west London. The laboratory served a population of 2.5 million. The research use of anonymised, 469 residual samples obtained for routine diagnostic purposes was approved by a research ethics committee 470 (IRAS Project ID 162013; REC reference 06/Q0406/20). In the period 2018-2019, 18 nitrofurantoinresistant $E$. coli isolates were collected. Nonetheless, the susceptibility result could be reproduced for 472 only nine of these isolates (IN01-09) when their frozen stock was retrieved and then retested using the 473 same disc diffusion method. Aerobic MIC of nitrofurantoin was measured for each of the nine isolates 474 using MIC Test Strips (Liofilchem ${ }^{\circledR}$, Italy) with nitrofurantoin concentrations ranging between 0.032 
$475512 \mathrm{mg} / \mathrm{L}$. Nitrofurantoin-susceptible E. coli isolate EC0098B (nitrofurantoin MIC: $32 \mathrm{mg} / \mathrm{L}$ ) was

476 chosen as a negative control. MICs were reported as per conventional two-fold series of concentration

477 increments $(2,4,8, \ldots \mathrm{mg} / \mathrm{L})$ and interpreted in accordance with EUCAST breakpoints v10.0 (49).

478 In order to identify genetic variants related to nitrofurantoin resistance using a comparative

479 approach, the NCBI Pathogen Detection portal (www.ncbi.nlm.nih.gov/pathogens/), NCTC 3000

480 Project, EnteroBase, NIHR HPRU isolate collection (BioProject accession: PRJEB20357; manuscript

481 in preparation), and literature were searched for $E$. coli genomes from isolates with known

482 nitrofurantoin susceptibility profiles $(7,33,34,50,51)$. Complete and draft genome assemblies were

483 downloaded from the NCBI nucleotide database and sequence reads of unassembled genomes were

484 downloaded from NCBI Sequence Read Archive (SRA). In total, 208 E. coli genomes were obtained 485 from the search, consisting of 196 genomes downloaded from the GenBank database 486 (www.ncbi.nlm.nih.gov/genbank/) and 12 genomes from clinical isolates collected from north west 487 London by the HPRU in 2015-2016.

\section{Whole-genome DNA extraction and sequencing}

489 Genomic DNA of isolates IN01-09 was extracted using a phenol/chloroform method (52), checked

490 for integrity using gel electrophoresis, and stored at $-20^{\circ} \mathrm{C}$. DNA libraries were prepared in a paired491 end layout with NEBNext ${ }^{\circledR}$ Ultra $^{\mathrm{TM}}$ II FS DNA Library Prep Kit (New England BioLabs, UK) and 492 sequenced under a 150-bp read length using Illumina HiSeq 4000 systems and HiSeq SBS Kit v4 493 reagents (Illumina, USA). Read demultiplexing and adapter trimming were performed by the Imperial 494 BRC Genomics Facility using their production pipeline.

\section{Quality control of sequence reads}

496 Quality summaries of all read files were generated using FastQC v0.11.9 and compiled using MultiQC 497 v1.8 $(53,54)$. With Trimmomatic v0.39, the reads of IN01-09 were trimmed for an average base 498 quality of Phred Q20 in a 5-bp sliding window and were then filtered for a minimum length of $50 \mathrm{bp}$ 
499 (55). Seqkit v0.12.0 was used for randomly sampling the filtered reads of each genome to an 80-fold

500 coverage, assuming an average genome size of $5 \mathrm{Mbp}(56,57)$. For reads downloaded from the SRA,

501 case-by-case read trimming and filtering was conducted using Trimmomatic in order to deal with large

502 variation in read quality and to obtain a coverage of less than 100 folds. Genomes having less than 40-

503 fold coverages were excluded from further analysis. DNA contamination in reads was evaluated for

504 each genome using Kraken v2.0.8-beta and its full bacterial database (accessed in February 2020) (58).

\section{De novo genome assembly and annotation}

506 Reads of each genome were assembled using Unicycler v0.4.9b (59). Quality of assemblies were

507 evaluated based on summary statistics calculated by QUAST v5.0.2 (60). In summary, 110-236

508 contigs (median: 137) and an assembly length of 4.6-5.3 Mbp (median: 4.9 Mbp) were obtained per

509 genome (Table S2). Complete plasmid sequences were identified in Bandage visualisation of assembly

510 graphs (61). Assemblies were annotated through Prokka v1.13 with a reference protein database

511 representing proteins extracted from all E. coli genomes that were publicly available in the NCBI

512 RefSeq database by March 2020 (62). Redundant protein sequences were removed from this database

513 using CD-HIT v4.8.1 (63) under a minimum amino acid identity of 70\%.

\section{Estimating population structure}

515 Multi-locus sequence typing (MLST) of genomes IN01-09 was conducted using ARIBA v2.14.4 and

516 the MLST of other collected genomes were conducted using mlst (github.com/tseemann/mlst) (64).

517 The Achtman scheme for E. coli, which was downloaded from PubMLST database (pubmlst.org) with

518 ARIBA in March 2020, was used for the MLST analysis. Core-genome population structure was

519 estimated through clustering all genome assemblies with PopPUNK v2.0.2 given k-mer lengths

520 increased from 15 bp to 23 bp by a 2-bp step size (23). 


\section{Identifying genetic alterations of $n f_{s} A, n f s B$, and $r i b E$}

522 For each of sample genomes IN01-09, the most closely related complete genome of a nitrofurantoin-

523 susceptible isolate was chosen from our collection as a reference according to core-genome distances

524 calculated by PopPUNK. Reads of the sample genome were then mapped to the chromosome sequence

525 of this reference genome using Bowtie v2.4.1 under the mode sensitive-local (65). Single-nucleotide

526 variants (SNVs) and indels were identified from mapped reads using BCFtools v1.9 when filtering out

527 low-confidence variant calls (QUAL $\leq 20, \mathrm{DP} \leq 10$, or MQ $\leq 20)$ as well as low-quality base calls (Phred

$528 \mathrm{Q}<20)$. Moreover, SNVs and indels within repetitive regions identified in the reference sequence with

529 nucmer v3.1 were excluded for accuracy (66). All remaining variants in the sample genome were

530 annotated using SnpEff v4.3t and a database built from the reference sequence (67).

Bandage v0.8.1 (61) was used for searching for loci of $n f s A, n f s B$, and $r i b E$ in genome assemblies

532 of IN01-09 and extracting allele sequences. SNVs and indels were identified in these alleles using

533 web-based megaBLAST (blast.ncbi.nlm.nih.gov) and compared to those identified through read

534 mapping. Interruptive insertion sequences were inferred from assembly graphs using Bandage and

535 were searched against the ISFinder database for reference sequences and classification (68). Insertion

536 sites of these putative insertion sequences were determined with ISMapper v2.0.1 (69). Copy numbers

537 of $n f_{s} A, n f_{s} B$, and $r i b E$ in each sample genome were predicted from mapped reads using CNOGpro

538 v1.1 (100-bp sliding windows and 1,000 bootstrap samples per gene) (70, 71). Translated sequences

539 of these three genes were extracted from Prokka annotations and were aligned using the ClustalW

540 algorithm implemented in MEGA X $(72,73)$. Functional effect of each amino acid substitution was

541 predicted using PROVEAN Protein (accessed in June 2020).

\section{$542 \quad$ Validating interrupted $n f s A$ and $n f s B$}

543 PCR experiments were conducted to confirm insertion sequences that might have interrupted $n f s A$ and

$544 n f s B$ in genomes IN01-03. Plausible sequence of each interrupted region was reconstructed from the 545 assembly graph using Bandage and thereby 22-bp PCR primers (Table 5) were designed using 
546 Primer3web v4.1.0 (primer3.ut.ee) (74). Primer positions in template genomic regions and expected

547 PCR products are illustrated in Fig. 2. PCR was carried out using a T100 ${ }^{\mathrm{TM}}$ Thermal Cycler (Bio-Rad,

548 USA) and GoTaq ${ }^{\circledR}$ DNA Polymerase (Promega, UK). PCR primers and products were sent to Genewiz

549 (UK) for Sanger sequencing. Reads were trimmed for removing ambiguous bases before alignment.

550 TABLE 5 PCR primers for validating interrupted genetic regions in genome IN01-03.

\begin{tabular}{|c|c|c|c|c|c|}
\hline Genome & Primer & Sequence & $\mathbf{T}_{\mathbf{m}}\left({ }^{\circ} \mathbf{C}\right)$ & $\mathbf{T}_{\mathbf{a}}\left({ }^{\circ} \mathbf{C}\right)$ & Product size (bp) \\
\hline \multirow[t]{4}{*}{ IN01, 02} & L1 & GTGGTGGTTATTCTTCAGGTGG & 64.3 & 59.3 & 974 \\
\hline & $\mathrm{R} 1$ & GAAGGGAAAGCTGCACGTAATC & 65.8 & & \\
\hline & L2 & CAGCTCCACCGATTTTGAGAAC & 66.9 & 61.7 & 814 \\
\hline & $\mathrm{R} 2$ & СААТTTTCAСССТGCАССТСТС & 66.7 & & \\
\hline \multirow[t]{6}{*}{ IN03 } & L1 & CGTCCTGACTCAACCGTAAATC & 64.5 & 58.7 & 895 \\
\hline & $\mathrm{R} 1$ & ССТААААТСТАСТCAGCGTCGG & 63.7 & & \\
\hline & L2 & GCTTGTCCCAACCTTGTTTCTG & 66.7 & 61.5 & 895 \\
\hline & $\mathrm{R} 2$ & CCAACCAAAGCGAGAACAAAAC & 66.5 & & \\
\hline & L3 & GAAGCTCGCAATACCATAAGCC & 65.6 & 60.6 & 954 \\
\hline & R3 & CATCGAGGTGGTGTGATCAATC & 66.6 & & \\
\hline
\end{tabular}

$551 \mathrm{~T}_{\mathrm{m}}$ : melting temperature; $\mathrm{T}_{\mathrm{a}}$ : annealing temperature. $\mathrm{L}$ and $\mathrm{R}$ in primer names refer to paired left and 552 right primers, respectively.

553 Detecting acquired antimicrobial resistance genes

554 An ARIBA-compatible reference database of acquired AMR genes was created from the ResFinder 555 database (commit hash: 7e1135b) through quality filtering and sequence clustering (nucleotide identity $556 \geq 80 \%)$ using ARIBA and CD-HIT-EST v4.8.1 (29, 63). Acquired AMR genes in genomes IN01-09 557 were detected from sequence reads using ARIBA and this reference database, whereas these genes in 558 other $E$. coli genomes were identified from genome assemblies using ABRicate 559 (github.com/tseemann/abricate) (75) and the ResFinder database without clustering.

560 Screening for nitrofurantoin-resistance determinants in the UK E. coli population

561 Genome assemblies of UK E. coli isolates were downloaded from NCBI nucleotide databases (as of 562 August 2020; Table S3) or retrieved from our ongoing NIHR HPRU study of E. coli bacteraemia and 563 intestinal colonisation. Collection dates and locations of isolates were retrieved from the NCBI 
564 BioSample database, NCTC Bacteria and Mycoplasmas Browse (www.phe-culturecollections.org.uk),

565 EnteroBase, and related literature. This information was manually inspected for accuracy and non566 redundancy. A non-redundant reference database NITREc was created from allelic and translated 567 sequences of $n f_{s} A, n f s B$, and $r i b E$ in all the 217 E. coli isolates of known nitrofurantoin susceptibility 568 (Fig. 1). Specifically, CD-HIT-EST and CD-HIT were used for sequence deduplication. Computer 569 scripts developed for gene detection and mutation identification are available in the NITREc code repository (github.com/wanyuac/NITREc/tree/master/Script) (including those mentioned below).

Nucleotide BLAST (megaBLAST) v2.9.0 (76) was used for identifying $n f s A, n f s B$, ribE, oqxA, and

$o q x B$ and for extracting their allele sequences (script screenGenes.pbs), which were then translated

573 into protein sequences using script translateDNA.py. Particularly, alleles of $n f s A, n f s B$, and $r i b E$ from

574 nitrofurantoin susceptible E. coli strain ATCC25922 and alleles of oqxA and oqxB from the ResFinder

575 database were used as queries for gene screen. Since missense mutations in the start or stop codon may

576 reduce the length of a BLAST alignment, hits showing partial or complete truncation of either codon

577 were manually verified. CD-HIT-EST and CD-HIT were used for identifying identical nucleotide and

578 protein sequences, respectively. Sequence alignments were generated using Clustal Omega

579 (www.ebi.ac.uk/Tools/msa/clustalo). Nonsense mutations were determined through comparing

580 lengths of predicted protein sequences to those of their reference sequences. Missense mutations were 581 identified in the alignments of protein sequences using script missenseFinder.py, which identifies 582 amino acid substitutions of each query protein sequence by comparing it to its most closely related 583 reference protein sequence in the NITREc database. Finally, using script findKnownMutations.py, 584 missense mutations were searched for those known to be associated with nitrofurantoin resistance 585 (Tables A1-3, and Table 1).

586 Evolutionary analysis of missense mutations in $n f_{s} A, n f s B$, and $r i b E$

587 An ML estimate of the ratio $\omega$ of each gene was estimated from the codon alignment using a reference588 free approach implemented in GenomegaMap v1.0.1 (77), assuming a constant $\omega$ per gene. 
589 Specifically, for each gene, full-length, indel-free alleles were translated into protein sequences using

590 NITREc script translateDNA.py (codon table: 11). Premature proteins (due to nonsense mutations)

591 and their corresponding alleles were identified and then excluded using NITREc script

592 rmProteinsByLength.py. A multi-sequence alignment of remaining alleles was generated using

593 program MUSCLE in software package MEGA X (78) and was then converted into a codon alignment

594 using script pal2nal.pl (79).

595 For each of genes $n f s A, n f s B$, and $r i b E$, SNP sites were identified in a deduplicated set of allele

596 sequences that were used for estimating the $\omega$ ratios - namely, untruncated alleles that only carried

597 missense mutations. A maximum-likelihood (ML) phylogenetic tree was reconstructed for each gene

598 from the alignment of deduplicated alleles. Specifically, nucleotide sequences were deduplicated using

599 CD-HIT-EST before tree reconstruction. An extended selection of substitution models by IQ-Tree

600 v1.6.12 (parameters: -t BIONJ -m MF -mtree) was performed for each gene (80). Since the best-fit

601 model differed between genes, candidate models of each gene were sorted in an ascending order of

602 models' Bayesian information criterion (BIC) scores, and the model consistently ranked $<10$ across

603 all three genes was chosen for the tree reconstruction. Then an ML tree was reconstructed for each

604 gene by IQ-Tree with the chosen model (TIM3e + R2), a BIONJ starting tree, 10 independent runs (for

605 selecting the tree of the greatest maximum likelihood), and 500 bootstrap replicates (for supporting

606 branches in the selected tree).

607 Identification of variant sites in the sequence alignment used for reconstructing each gene tree was

608 performed with snp-sites v2.5.1 (81). Homoplasy sites in the alignment were identified and plotted

609 using R package homoplasyFinder (82), which took as input the gene tree in addition to the allele

610 alignment. A PHI was calculated from the allele alignment of each gene and the significance of PHI

611 was tested for using PHIPack (83) with a 80-bp sliding window, given the null hypothesis of no

612 recombination. Recombination within a gene was considered significant if the p-value of a PHI did

613 not exceed 0.05 . 


\section{Predicting nitrofurantoin susceptibility from detected genetic diversity}

615 The prediction of nitrofurantoin susceptibility was conducted independently for each HPRU isolate at 616 sequence, genetic, and genomic levels. It also considered whether the gene of interest is intrinsic or 617 acquired. The prediction method can be concisely described using the following scoring algorithm, 618 which assumes that loss of intrinsic gene function and gain of both functional oqxA and oqxB alleles 619 are associated with reduced nitrofurantoin susceptibility. Calculation of gene-level and genome-level 620 scores have been implemented in NITREc helper script scoreHitsNITR.R, whereas manual inspections 621 are required to determine sequence-level scores.

Firstly, at the sequence level, supposing $n_{i}$ BLAST hits of gene $i(i=n f s A, n f s B$, ribE, oqxA, or 624 probability of this sequence to confer reduced nitrofurantoin susceptibility. Then an arbitrary value is assigned to the score for each hit of intrinsic genes: $s_{i j}=1$, if the sequence is truncated or interrupted (which cannot be distinguished from each other by BLAST without assembly graphs) or has a startcodon loss, a frameshift or nonsense mutation, or any missense mutation known to be associated with nitrofurantoin resistance; $s_{i j}=0.1$, if the sequence only has missense mutations whose impact on 629 nitrofurantoin susceptibility remains unknown; and $s_{i j}=0$, if the sequence is identical to any known 630 "wildtype" allele present in a nitrofurantoin-susceptible isolate. On the other hand, for each hit of acquired genes, $s_{i j}=1$, if the sequence is identical to a reference allele in the ResFinder database; $s_{i j}=$ $s_{i j}=0$, if the sequence is truncated or interrupted (No other kind of $o q x A$ or $o q x B$ variants were found 634 in this study).

Secondly, a gene score $g_{i}(i=n f s A, n f s B, \operatorname{ribE}, o q x A, o q x B)$ is determined from sequence scores. 
reduced nitrofurantoin susceptibility. Moreover, $g_{i}=1$ and $g_{i}{ }^{\prime}=0$ when intrinsic gene $i$ and acquired

640 gene $i$ ' are not detected, respectively.

641 Finally, a risk score $r$ is calculated from gene scores for each genome. Let $g_{1}, \ldots, g_{5}$ represent gene

642 scores of $n f s A, n f s B$, ribE, oqxA, and $o q x B$, respectively, then each gene score takes a value of $0,0.1$,

643 or 1. Since the multidrug effulx pump OqxAB requires both functional components OqxA and OqxB

644 to reduce the intracellular concentration of nitrofurantoin, the risk score is calculated by formula:

$$
r=\sum_{i=1}^{3} g_{i}+\min \left(g_{4}, g_{5}\right)
$$

Hence $r$ takes values between zero (nitrofurantoin susceptible) and four (nitrofurantoin resistant).

647 In our study, genomes were sorted by risk scores to identify plausible resistant and susceptible isolates.

648 Predicted nitrofurantoin susceptibility was labelled and interpreted in accordance with the EUCAST

649 terminology: S (susceptible, standard dosing regimen), if $r=0$; I (susceptible, increased exposure), if

$r=1$; R (resistant), if $r \geq 2$; S/I, if $r=0.1$; and S/I/R, if $0.1<r<1$ or $1<r<2$.

\section{Validating predictions of nitrofurantoin resistance}

652 Since HPRU isolates of known nitrofurantoin susceptibility (based on clinical records) had been used

653 by the prediction algorithm as references, we retrieved HPRU isolates of unknown nitrofurantoin 654 susceptibility for validating the predictions. Nitrofurantoin MICs were determined using Etests 655 (Liofilchem ${ }^{\circledR}$ MIC Test Strips). First, 20 isolates that were anticipated to show reduced nitrofurantoin susceptibility were selected for Etests (Tables 3 and 4). Second, additional seven HPRU isolates were included for Etests because these isolates had mutations detected in $n f s A$ or $n f s B$ of IN08, or carried an alternative stop codon or novel mutation in $r i b E$, although the predicted susceptibility was uncertain

659 due to a lack of information from literature (Table 4). Furthermore, disc diffusion tests (as per 660 EUCAST manual v8.0) (84) and MALDI-TOF mass spectrometry were conducted for confirming the 661 heterogeneous responses to nitrofurantoin. A positive control (IN09) and a negative control 662 (ATCC25922) were included for experiments. (Doc. S1) 


\section{Data availability}

664 Sequence reads and genome assemblies of isolates IN01-09 have been deposited in the European

665 Nucleotide Archive (ENA) under BioProject accession number PRJEB38850. Nucleotide and protein 666 sequences of $n f s A, n f s B$, and $r i b E$ alleles in genomes of these nine isolates can be accessed in our 667 database NITREc. The database also offers nucleotide sequences of genetic elements related to the 668 interrupted $n f s A / n f s B$ regions in IN01-03 (github.com/wanyuac/NITREc/tree/master/Seq/Nucl/IS). 669 All supplementary files are available on figshare (doi.org/10.6084/m9.figshare.14632653.v1).

\section{Supplementary materials}

TABLE S1, Excel file. Sample information and ENA accessions of E. coli isolates IN01-09.

Bacterial susceptibility to trimethoprim, ciprofloxacin, gentamicin, co-amoxiclav, cephalexin, amoxicillin, amikacin, meropenem, cefoxitin, ceftazidime, piperacillin-tazobactam, cefotaxime, mecillinam, ceftazidime-avibactam, ertapenem, temocillin, and fosfomycin was automatically tested for by the same hospital microbiology laboratory using the EUCAST disc diffusion method.

TABLE S2, Excel file. Supplementary results from analysing genomes of isolates IN01-09.

TABLE S3, Excel file. Accessions and sample information of public genome assemblies used for

E. coli genomes.

TABLE S5, Excel file. Genotypes and predicted nitrofurantoin susceptibility of 69 HPRU E. coli isolates that were used for the validation/exploratory experiment.

FIG S1, PDF image. Homoplasy detected in $n f s A$ alleles by homoplasyFinder. Left: an ML, 
are labelled by homoplasy-SNP positions deduced through ancestral reconstruction. Right: Genotype of the matrix.

FIG S2, PDF image. Homoplasy detected in representative $n f s B$ alleles by homoplasyFinder. Left: an ML, midpoint-rooted, and ladderised phylogenetic tree of $231 n f s B$ alleles (654 bp, no nonsense 692 mutation) identified in 12,319 BLAST hits of $n f_{s} B$ (Fig. 3) in our collection of UK E. coli genomes. 693 Tips of the tree are labelled by names of representative genomes where the alleles were present. 694 Internal nodes are labelled by homoplasy-SNP positions deduced through ancestral reconstruction. 695 Right: Genotype matrix of 50 homoplasy SNP sites in these $n f s B$ alleles. Positions of these sites are 696 labelled at the top of the matrix.

FIG S3, PDF image. Homoplasy detected in representative ribE alleles by homoplasyFinder. Left: an ML, midpoint-rooted, and ladderised phylogenetic tree of $88 \mathrm{ribE}$ alleles (471 bp, no nonsense mutation) identified in 12,407 BLAST hits of ribE (Fig. 3) in our collection of UK E. coli genomes.

Tips of the tree are labelled by names of representative genomes where the alleles were present. Internal nodes are labelled by homoplasy-SNP positions deduced through ancestral reconstruction. Right: Genotype matrix of 12 homoplasy SNP sites in these ribE alleles. Positions of these sites are labelled at the top of the matrix.

DATASET S1, 7-Zip compressed directory consisting of assembly graphs of genomes IN01-09 and the ML phylogenetic trees of $n f s A, n f s B$, and $r i b E$.

DOC S1, PDF document. Methods for the experimental validation of predicted nitrofurantoin 708 susceptibility and the identification of nitrofurantoin-resistant subpopulations of heterogeneous $E$. coli isolates. 


\section{Funding}

710 This research was funded by the National Institute for Health Research (NIHR) Health Protection

711 Research Unit (HPRU) in Healthcare Associated Infections and Antimicrobial Resistance at Imperial

712 College London in partnership with Public Health England (PHE). The views expressed in this

713 publication are those of the author(s) and not necessarily those of the NHS, the National Institute for

714 Health Research, the Department of Health and Social Care or Public Health England.

\section{Acknowledgments}

716 The authors acknowledge the support of the National Institute for Health Research (NIHR) Imperial

717 College Biomedical Research Centre (BRC), which funds the BRC Infection Bioresource, the Imperial

718 BRC Genomics Facility, and the Colebrook Laboratory. The Imperial BRC Genomics Facility has

719 provided resources and support that have contributed to the research results reported within this paper.

720 We are also grateful to the staff in the Imperial College NHS Healthcare Trust Diagnostic Laboratory

721 for their generous support and expertise.

We thank Ho Kwong Li, Kristin Krohn Huse, Max Pearson, Matthew K. Siggins, Hanqi Li

723 (Imperial College London), and Rūta Prakapaitè (the University of Basel) for sharing experimental

724 expertise. We express our gratitude to John A. Lees (Imperial College London) for advice on using 725 software PopPUNK.

726 EJ is a Rosetrees/Stoneygrate 2017 Imperial College Research Fellow, funded by Rosetrees Trust 727 and the Stoneygate Trust (Reference number: M683). NJC was supported by a Sir Henry Dale 728 Fellowship, jointly funded by the Wellcome Trust and Royal Society (104169/Z/14/A), and a Medical

729 Research Council (MRC) grant (MR/T016434/1). YW was partly supported by the NIHR Senior 730 Investigator Award of Professor Alison Holmes (Imperial College London) and by the Imperial 731 College Biomedical Research Centre. 


\section{Authors' contributions}

733 Experimental design, primary genomic analysis, prediction algorithm: YW; Laboratory work: EM,

734 RCYL, XZ, YW; Comparative genomics, evolutionary analyses, and interpretation: AV, EJ, NJC, and

735 YW; Project conception: SS, MJE, and NW; Project supervision: SS and MJE; First draft of the 736 manuscript: YW; Manuscript revisions: SS, EJ, AV, NJC, MJE, NW, and RCYL; Final manuscript 737 editing and approval: all authors.

\section{Competing interests}

739 The authors declare that they have no competing interests.

\section{Appendices}

741 In this section, we list known missense mutations in $\mathrm{NfsA}, \mathrm{NfsB}$, and RibE that were exclusively

742 identified in E. coli isolates of reduced nitrofurantoin susceptibility. Particularly, we only consider

743 experimentally confirmed (such as using stepwise mutations) causative mutations and those having a

744 deleterious impact on protein structure or translation by prediction. Absence of these mutations in

745 known nitrofuranoin-susceptible $E$. coli was confirmed through aligning wildtype protein sequences

746 from the NITREc database with MEGA X.

747 TABLE A1 NfsA missense mutations associated with reduced nitrofurantoin susceptibility in E. coli.

\begin{tabular}{lllllll}
\hline Pos. & Ref. & Alt. & Ref. accession & Alt. accession & Confidence & Citation \\
\hline 1 & M & I & NG & NA & Possible reduced translation & $(35)$ \\
11 & H & Y & WP_000189141.1 & QEL51189.1 & PROVEAN prediction & $(36)$ \\
11 & H & Y & AAC73938.1 & WP_119187568.1 & PROVEAN prediction & $(85)$ \\
11 & H & L & AAC73938.1* & NA & Experimentally confirmed & $(6)$ \\
15 & R & C & NG & NA & Experimentally confirmed & $(35,41)$ \\
33 & S & R & AAC73938.1 & WP_094323839.1 & PROVEAN prediction & $(35,85)$ \\
33 & S & N & WP_000189165.1 & QEL51188.1 & PROVEAN prediction & $(36)$ \\
36 & A & V & NG & NA & PROVEAN prediction & $(41)$ \\
38 & S & Y & WP_000189167.1 & QEL51184.1 & PROVEAN prediction & $(36)$ \\
67 & Q & L & AAC73938.1 & NA & PROVEAN prediction & $(85)$ \\
80 & C & R & AAC73938.1 & NA & PROVEAN prediction & $(85)$ \\
89 & I & N & AAC73938.1* & NA & Experimentally confirmed & $(6)$
\end{tabular}




\begin{tabular}{lllllll}
126 & G & R & AAC73938.1 & WP_023149811.1 & PROVEAN prediction & $(85)$ \\
131 & G & D & NG & NA & Previously reported & $(35)$ \\
133 & R & S & AAC73938.1\# & WP_096948202.1 & In active-site pocket & $(20)$ \\
154 & G & E & AAC73938.1 & WP_000189151.1 & PROVEAN prediction & $(85)$ \\
158 & G & C & NG & NA & PROVEAN prediction & $(41)$ \\
203 & R & L/A & NG & NA & Experimentally confirmed & $(35,37)$ \\
203 & R & C & AAC73938.1 & WP_072644388.1 & Experimentally confirmed & $(20,35$, \\
& & & & & & $37,85)$ \\
$\mathbf{2 1 2}$ & W & R & WP_000189134.1 & QEL51185.1 & PROVEAN prediction & $(36)$ \\
\hline
\end{tabular}

748 Mutations identified in IN01-09 are highlighted in boldface. Pos: Position; Ref: reference amino acid;

749 Alt: alternative amino acid; Ref. accession: NCBI accession of the reference protein sequence; Alt.

750 accession: NCBI accession of the mutant protein sequence; NG: not given in the cited literature; NA,

751 not available. * Based on the nucleotide sequence offered in Fig. 1 of literature (6), we confirmed that

752 the reference $n f s A$ allele in E. coli strain K-12 sub-strain AB1157 was identical to that in K-12 sub-

753 strain MG1655, hence we use the protein sequence (NCBI protein accession: AAC73938.1) from

754 MG1655 for the reference. No exact match of either mutant protein (carrying mutation H11L or I89N)

755 was found in the NCBI protein database for E. coli (accessed in August 2020). Two mutations (R15C

756 and Q67P) from Table 2 of literature (6) are not included here as we could not solve their discrepancy

757 to mutations illustrated in Fig. 1 of the same article. ${ }^{+}$Reference sequence was restored from mutants

758 and searched against the NCBI protein database (accessed in August 2020) for perfect matches. ${ }^{\#}$ This

$759 n f s A$ allele is identical to that in K-12 sub-strain MG1655. Hence the accession given by Ho, et al. in

760 their article (20), EIE36436.1, refers to an identical protein referred to by accession AAC73938.1.

$761 \$$ Identical protein found in the NCBI protein database, accessed in September 2020.

762 TABLE A2 NfsB missense mutations associated with reduced nitrofurantoin susceptibility in E. coli.

\begin{tabular}{lllllll}
\hline Pos. & Ref. & Alt. & Ref. accession & Alt. accession & Confidence & Citation \\
\hline 1 & M & I & NG & NA & Possible reduced translation & $(35)$ \\
33 & L & P & AAC73679.1* & NA & Experimentally confirmed & $(6)$ \\
34 & L & R & AAC73679.1* & WP_096943843.1 & Experimentally confirmed & $(6)$ \\
37 & S & R & AAC73679.1* & NA & Experimentally confirmed & $(6)$ \\
40 & S & C & AAC73679.1* & NA & Experimentally confirmed & $(6)$ \\
44 & Q & L & NG & NA & Experimentally confirmed & $(35)$ \\
44 & Q & P & AAC73679.1* & NA & Experimentally confirmed & $(6)$ \\
44 & Q & H & AAC73679.1 & EFN8391251.1 & PROVEAN prediction & $(85)$
\end{tabular}




\begin{tabular}{lllllll}
45 & P & S & WP_000351450.1 & QEL51196.1 & PROVEAN prediction & $(36)$ \\
45 & P & Q & AAC73679.1* & NA & Experimentally confirmed & $(6)$ \\
71 & N & K & AAC73679.1* & NA & Experimentally confirmed & $(6)$ \\
84 & F & S & WP_000351450.1 & QEL51192.1 & PROVEAN prediction & $(36)$ \\
84 & F & S & AAC73679.1 & NA & PROVEAN prediction & $(85)$ \\
93 & V & G & NG & NA & Experimentally confirmed & $(35)$ \\
105 & D & Y & AAC73679.1* & NA & Experimentally confirmed & $(6)$ \\
107 & R & H & NG & NA & Experimentally confirmed & $(35)$ \\
107 & R & C & AAC73679.1 & WP_096996177.1 & PROVEAN prediction & $(85)$ \\
138 & W & C & NG & NA & Experimentally confirmed & $(35)$ \\
148 & G & S & AAC73679.1* & NA & Experimentally confirmed & $(6)$ \\
165 & E & A & NG & NA & Experimentally confirmed & $(35)$ \\
166 & G & D & NG & NA & Experimentally confirmed & $(35)$ \\
$\mathbf{1 9 2}$ & G & D/A & NG & NA & Experimentally confirmed & $(35)$ \\
192 & G & S & AAC73679.1 & NA & PROVEAN prediction & $(85)$ \\
207 & R & H & AAC73679.1* & NA & Experimentally confirmed & $(6,85)$ \\
\hline
\end{tabular}

763 Mutations identified in IN01-09 are highlighted in boldface. Pos: Position; Ref: reference amino acid;

764 Alt: alternative amino acid; Ref. accession: NCBI accession of the reference protein sequence; Alt.

765 accession: NCBI accession of the mutant protein sequence; NG: not given in the cited literature; NA,

766 not available. * Based on the nucleotide sequence offered in Fig. 2 of literature (6), we confirmed that

767 the reference $n f s B$ allele in $E$. coli strain K-12 sub-strain AB1157 was identical to that in K-12 sub-

768 strain MG1655, hence we use the protein sequence (NCBI protein accession: AAC73679.1) from

769 MG1655 for the reference. An exact match to the protein harbouring mutation L34R was identified in

770 the NCBI protein database for E. coli, although no exact match to the mutant DNA sequence was found

771 in GenBank. Four mutations (S39C, V83D, Y183D, and V187E) from Table 2 of literature (6) are not

772 included here as we could not solve their discrepancy to mutations illustrated in Fig. 2 of the same

773 article. ${ }^{+}$A reference protein sequence was restored from both mutants and it completely matched to

774 WP_000351450.1 in the NCBI protein database. Database searches were performed in August 2020.

775 \$ Identical protein found in the NCBI protein database, accessed in September 2020.

776 TABLE A3 RibE missense mutations associated with reduced nitrofurantoin susceptibility in E. coli.

\begin{tabular}{lllllll}
\hline Pos. & Ref. & Alt. & Ref. accession & Alt. accession & Confidence & Citation \\
\hline 55 & P & H & WP_001021161.1 & NA & PROVEAN prediction & $(85)$ \\
85 & G & C & WP_001021161.1 & QEL51191.1 & PROVEAN prediction & $(36)$ \\
\hline
\end{tabular}


Pos: Position; Ref: reference amino acid; Alt: alternative amino acid; Ref. accession: NCBI accession of the reference protein sequence; Alt. accession: NCBI accession of the mutant protein sequence; NG: not given by the cited literature; NA, not available. ${ }^{*}$ Reference sequence was restored from the mutant and searched against the NCBI protein database (accessed in August 2020) for perfect matches.

\section{References}

782 1. Waisbren B, Crowley W. 1955. Nitrofurantoin: Clinical and laboratory evaluation. AMA Arch Intern Med 95:653-661.

2. Nickel JC. 2005. Management of Urinary Tract Infections: Historical Perspective and Current Strategies: Part 1—Before Antibiotics. J Urol 173:21-26.

786 3. Cunha BA. 1988. Nitrofurantoin-current concepts. Urology 32:67-71.

787 4. Garau J. 2008. Other antimicrobials of interest in the era of extended-spectrum beta-lactamases: fosfomycin, nitrofurantoin and tigecycline. Clin Microbiol Infect 14:198-202.

5. McOsker CC, Fitzpatrick PM. 1994. Nitrofurantoin: Mechanism of action and implications for resistance development in common uropathogens. J Antimicrob Chemother 33:23-30.

6. Whiteway J, Koziarz P, Veall J, Sandhu N, Kumar P, Hoecher B, Lambert IB. 1998. OxygenInsensitive Nitroreductases: Analysis of the Roles of $n f s A$ and $n f s B$ in Development of Resistance to 5-Nitrofuran Derivatives in Escherichia coli. J Bacteriol 180:5529-5539.

798 8. Foxman B. 2010. The epidemiology of urinary tract infection. Nat Rev Urol 7:653-660.

799 9. McNally A, Kallonen T, Connor C, Abudahab K, Aanensen DM, Horner C, Peacock SJ, Parkhill 800 J, Croucher NJ, Corander J. 2019. Diversification of Colonization Factors in a Multidrug-Resistant 
Escherichia coli Lineage Evolving under Negative Frequency-Dependent Selection. MBio 10:e00644-19.

10. Public Health England. 2019. English surveillance programme for antimicrobial utilisation and resistance (ESPAUR) report 2018 to 2019. London.

11. Giske CG. 2015. Contemporary resistance trends and mechanisms for the old antibiotics colistin, temocillin, fosfomycin, mecillinam and nitrofurantoin. Clin Microbiol Infect 21:899-905.

12. Honsbeek M, Tjon-A-Tsien A, Stobberingh E, de Steenwinkel J, Melles DC, Lous J, Richardus JH, Voeten H. 2020. Low antimicrobial resistance in general practice patients in Rotterdam, the city with the largest proportion of immigrants in the Netherlands. Eur J Clin Microbiol Infect Dis 39:929-935.

13. Barišić Z, Babić-Erceg A, Borzić E, Zoranić V, Kaliterna V, Carev M. 2003. Urinary tract infections in South Croatia: aetiology and antimicrobial resistance. Int J Antimicrob Agents 22:61-

14. Caracciolo A, Bettinelli A, Bonato C, Isimbaldi C, Tagliabue A, Longoni L, Bianchetti MG. 2011.

16. Bean DC, Krahe D, Wareham DW. 2008. Antimicrobial resistance in community and nosocomial Escherichia coli urinary tract isolates, London 2005 - 2006. Ann Clin Microbiol Antimicrob 7:13.

17. Blandy O, Honeyford K, Gharbi M, Thomas A, Ramzan F, Ellington MJ, Hope R, Holmes AH, Johnson AP, Aylin P, Woodford N, Sriskandan S. 2019. Factors that impact on the burden of Escherichia coli bacteraemia: multivariable regression analysis of 2011-2015 data from West London. J Hosp Infect 101:120-128. 
18. Public Health England. 2016. English surveillance programme for antimicrobial utilisation and resistance (ESPAUR) Report 2016.

19. Linkevicius M, Sandegren L, Andersson DI. 2013. Mechanisms and fitness costs of tigecycline resistance in Escherichia coli. J Antimicrob Chemother 68:2809-2819.

830 20. Ho P-L, Ng K-Y, Lo W-U, Law PY, Lai EL-Y, Wang Y, Chow K-H. 2015. Plasmid-Mediated OqxAB Is an Important Mechanism for Nitrofurantoin Resistance in Escherichia coli. Antimicrob Agents Chemother 60:537-543.

21. Jenkins ST, Bennett PM. 1976. Effect of Mutations in Deoxyribonucleic Acid Repair Pathways on the Sensitivity of Escherichia coli K-12 Strains to Nitrofurantoin. J Bacteriol 125:1214 LP 1216.

22. Obaseiki-Ebor EE. 1984. Resistance to nitrofurantoin and UV-irradiation in recA; $u v r A$; and $u v r A$, lexA, Escherichia coli mutants conferred by an R-plasmid from an Escherichia coli clinical isolate.

23. Lees JA, Harris SR, Tonkin-Hill G, Gladstone RA, Lo SW, Weiser JN, Corander J, Bentley SD, Mutat Res 139:5-8. Croucher NJ. 2019. Fast and flexible bacterial genomic epidemiology with PopPUNK. Genome Res 29:304-316.

24. Argimón S, Abudahab K, Goater RJE, Fedosejev A, Bhai J, Glasner C, Feil EJ, Holden MTG, Yeats CA, Grundmann H, Spratt BG, Aanensen DM. 2016. Microreact: visualizing and sharing data for genomic epidemiology and phylogeography. Microb Genomics 2:e000093.

25. Choi Y, Chan AP. 2015. PROVEAN web server: a tool to predict the functional effect of amino acid substitutions and indels. Bioinformatics 2015/04/06. 31:2745-2747.

26. Bolmström A, Arvidson S, Ericsson M, Karlsson A. 1988. A novel technique for direct quantification of antimicrobial susceptibility of microorganisms, abstr. 1209, p. 325. In Program and abstracts of the 28th Interscience Conference on Antimicrobial Agents and Chemotherapy. American Society for Microbiology, Washington, DC. 
27. Bankevich A, Nurk S, Antipov D, Gurevich AA, Dvorkin M, Kulikov AS, Lesin VM, Nikolenko SI, Pham S, Prjibelski AD, Pyshkin A V, Sirotkin A V, Vyahhi N, Tesler G, Alekseyev MA, Pevzner PA. 2012. SPAdes: a new genome assembly algorithm and its applications to single-cell sequencing. J Comput Biol A J Comput Mol Cell Biol 19:455-477.

28. Chalmers R, Sewitz S, Lipkow K, Crellin P. 2000. Complete Nucleotide Sequence of Tn10. J Bacteriol 182:2970 LP - 2972.

29. Zankari E, Hasman H, Cosentino S, Vestergaard M, Rasmussen S, Lund O, Aarestrup FM, Larsen M V. 2012. Identification of acquired antimicrobial resistance genes. J Antimicrob Chemother 2012/07/12. 67:2640-2644.

30. Kallonen T, Brodrick HJ, Harris SR, Corander J, Brown NM, Martin V, Peacock SJ, Parkhill J. 2017. Systematic longitudinal survey of invasive Escherichia coli in England demonstrates a stable population structure only transiently disturbed by the emergence of ST131. Genome Res 27:1437-1449.

31. Brodrick HJ, Raven KE, Kallonen T, Jamrozy D, Blane B, Brown NM, Martin V, Török ME, Parkhill J, Peacock SJ. 2017. Longitudinal genomic surveillance of multidrug-resistant Escherichia coli carriage in a long-term care facility in the United Kingdom. Genome Med 9:70.

32. Goswami C, Fox S, Holden M, Connor M, Leanord A, Evans TJ. 2018. Genetic analysis of invasive Escherichia coli in Scotland reveals determinants of healthcare-associated versus community-acquired infections. Microb Genomics 4.

33. Public Health England. NCTC 3000 project: A comprehensive resource of bacterial type and reference genomes.

34. Zhou Z, Alikhan N-F, Mohamed K, Fan Y, Group the AS, Achtman M. 2020. The EnteroBase user's guide, with case studies on Salmonella transmissions, Yersinia pestis phylogeny, and Escherichia core genomic diversity. Genome Res 30:138-152.

35. Sandegren L, Lindqvist A, Kahlmeter G, Andersson DI. 2008. Nitrofurantoin resistance 
mechanism and fitness cost in Escherichia coli. J Antimicrob Chemother 62:495-503. epidemiology and nitrofurantoin resistance determinants from nitrofurantoin non-susceptible Escherichia coli isolated from urinary tract infections. J Glob Antimicrob Resist 21:335-339.

37. Kobori T, Sasaki H, Lee WC, Zenno S, Saigo K, Murphy MEP, Tanokura M. 2001. Structure and

39. Kahlmeter G. 2000. The ECO•SENS Project: a prospective, multinational, multicentre

38. Yang J, Zhan J, Bai J, Liu P, Xue Y, Yang Q. 2013. Residue Phe42 is critical for the catalytic site-directed mutagenesis of a flavoprotein from Escherichia coli that reduces nitrocompounds: alteration of pyridine nucleotide binding by a single amino acid substitution. $J$ Biol Chem 276:2816-2823.

activity of Escherichia coli major nitroreductase NfsA. Biotechnol Lett 35:1693-1700. epidemiological survey of the prevalence and antimicrobial susceptibility of urinary tract pathogens-interim report. J Antimicrob Chemother 46:15-22.

40. Race PR, Lovering AL, Green RM, Ossor A, White SA, Searle PF, Wrighton CJ, Hyde EI. 2005. Structural and mechanistic studies of Escherichia coli nitroreductase with the antibiotic nitrofurazone: Reversed binding orientations in different redox states of the enzyme. J Biol Chem 280:13256-13264.

41. Zhang X, Zhang Y, Wang F, Wang C, Chen L, Liu H, Lu H, Wen H, Zhou T. 2018. Unravelling mechanisms of nitrofurantoin resistance and epidemiological characteristics among Escherichia coli clinical isolates. Int J Antimicrob Agents 52:226-232.

42. Meyer J, Iida S, Arber W. 1980. Does the insertion element IS 1 transpose preferentially into A+Trich DNA segments? Mol Gen Genet MGG 178:471-473.

43. Foster TJ, Davis MA, Roberts DE, Takeshita K, Kleckner N. 1981. Genetic organization of transposon Tn10. Cell 23:201-213.

44. Hawkey J, Monk JM, Billman-Jacobe H, Palsson B, Holt KE. 2020. Impact of insertion sequences 
on convergent evolution of Shigella species. PLOS Genet 16:e1008931.

902 45. Yamazaki Y, Niki H, Kato J. 2008. Profiling of Escherichia coli Chromosome database, p. 385389. In Microbial Gene Essentiality: Protocols and Bioinformatics. Springer.

904 46. Jordan IK, Rogozin IB, Wolf YI, Koonin E V. 2002. Essential Genes Are More Evolutionarily 905 Conserved Than Are Nonessential Genes in Bacteria. Genome Res 12:962-968.

906 47. Rocha EPC, Danchin A. 2004. An Analysis of Determinants of Amino Acids Substitution Rates in Bacterial Proteins. Mol Biol Evol 21:108-116.

48. Poulsen HO, Johansson A, Granholm S, Kahlmeter G, Sundqvist M. 2013. High genetic diversity of nitrofurantoin- or mecillinam-resistant Escherichia coli indicates low propensity for clonal

49. European Committee on Antimicrobial Susceptibility Testing. 2020. Breakpoint tables for

913 50. Xavier BB, Vervoort J, Stewardson A, Adriaenssens N, Coenen S, Harbarth S, Goossens H, Malhotra-Kumar S. 2014. Complete Genome Sequences of Nitrofurantoin-Sensitive and -

52. He F. 2011. E. coli Genomic DNA Extraction. Bio-protocol 1:e97.

919 53. Andrews S. 2010. FastQC: A quality control tool for high throughput sequence data. Ref Source. 920 54. Ewels P, Magnusson M, Lundin S, Käller M. 2016. MultiQC: summarize analysis results for

55. Bolger AM, Lohse M, Usadel B. 2014. Trimmomatic: a flexible trimmer for Illumina sequence

924 56. Shen W, Le S, Li Y, Hu F. 2016. SeqKit: A Cross-Platform and Ultrafast Toolkit for FASTA/Q $925 \quad$ File Manipulation. PLoS One 11:e0163962. 
57. Touchon M, Perrin A, de Sousa JAM, Vangchhia B, Burn S, O’Brien CL, Denamur E, Gordon D, Rocha EPC. 2020. Phylogenetic background and habitat drive the genetic diversification of Escherichia coli. PLOS Genetics 16(6): e1008866.

58. Wood DE, Lu J, Langmead B. 2019. Improved metagenomic analysis with Kraken 2. Genome Biol 20:257.

59. Wick RR, Judd LM, Gorrie CL, Holt KE. 2017. Unicycler: Resolving bacterial genome assemblies from short and long sequencing reads. PLOS Comput Biol 13:e1005595.

60. Gurevich A, Saveliev V, Vyahhi N, Tesler G. 2013. QUAST: quality assessment tool for genome assemblies. Bioinformatics 29:1072-1075.

61. Wick RR, Schultz MB, Zobel J, Holt KE. 2015. Bandage: interactive visualization of de novo genome assemblies. Bioinformatics 31:3350-3352.

62. Seemann T. 2014. Prokka: rapid prokaryotic genome annotation. Bioinformatics 30:2068-2069.

63. Li W, Godzik A. 2006. Cd-hit: a fast program for clustering and comparing large sets of protein or nucleotide sequences. Bioinformatics 22:1658-1659.

940 64. Hunt M, Mather AE, Sánchez-Busó L, Page AJ, Parkhill J, Keane JA, Harris SR. 2017. ARIBA:

65. Langmead B, Salzberg SL. 2012. Fast gapped-read alignment with Bowtie 2. Nat Meth 9:357359.

66. Kurtz S, Phillippy A, Delcher AL, Smoot M, Shumway M, Antonescu C, Salzberg SL. 2004. Versatile and open software for comparing large genomes. GENOME Biol 5.

67. Cingolani P, Platts A, Wang LL, Coon M, Nguyen T, Wang L, Land SJ, Lu X, Ruden DM. 2012. A program for annotating and predicting the effects of single nucleotide polymorphisms, SnpEff. Fly (Austin) 6:80-92.

68. Siguier P, Perochon J, Lestrade L, Mahillon J, Chandler M. 2006. ISfinder: the reference centre for bacterial insertion sequences. Nucleic Acids Res 34 . 
69. Hawkey J, Hamidian M, Wick RR, Edwards DJ, Billman-Jacobe H, Hall RM, Holt KE. 2015. ISMapper: identifying transposase insertion sites in bacterial genomes from short read sequence data. BMC Genomics 16:1-11.

954 70. Brynildsrud O, Snipen L-G, Bohlin J. 2015. CNOGpro: detection and quantification of CNVs in 955 prokaryotic whole-genome sequencing data. Bioinformatics 31:1708-1715.

956 71. Brynildsrud O. 2018. Read Depth Analysis to Identify CNV in Bacteria Using CNOGpro, p. 73957 81. In Bickhart, DM (ed.), Copy Number Variants: Methods and Protocols. Springer New York, $958 \quad$ New York, NY.

959 72. Thompson JD, Gibson TJ, Higgins DG. 2003. Multiple Sequence Alignment Using ClustalW and $960 \quad$ ClustalX. Curr Protoc Bioinforma 00:2.3.1-2.3.22.

961 73. Kumar S, Stecher G, Li M, Knyaz C, Tamura K. 2018. MEGA X: Molecular Evolutionary 962 Genetics Analysis across Computing Platforms. Mol Biol Evol 35:1547-1549.

963 74. Untergasser A, Cutcutache I, Koressaar T, Ye J, Faircloth BC, Remm M, Rozen SG. 2012. 964 Primer3 - new capabilities and interfaces. Nucleic Acids Res 40:e115-e115.

965 75. Seemann T. Abricate. GitHub.

966 76. Camacho C, Coulouris G, Avagyan V, Ma N, Papadopoulos J, Bealer K. 2009. BLAST+: $967 \quad$ architecture and applications. BMC Bioinformatics 10.

968 77. Wilson DJ. 2020. GenomegaMap: Within-Species Genome-Wide dN/dS Estimation from over 969 10,000 Genomes. Mol Biol Evol 37:2450-2460.

970 78. Edgar RC. 2004. MUSCLE: multiple sequence alignment with high accuracy and high throughput. $971 \quad$ Nucleic Acids Res 32:1792-1797.

972 79. Suyama M, Torrents D, Bork P. 2006. PAL2NAL: robust conversion of protein sequence 973 alignments into the corresponding codon alignments. Nucleic Acids Res 34:W609-W612.

974 80. Nguyen L-T, Schmidt HA, von Haeseler A, Minh BQ. 2015. IQ-TREE: A Fast and Effective 975 Stochastic Algorithm for Estimating Maximum-Likelihood Phylogenies. Mol Biol Evol 32:268- 
bioRxiv preprint doi: https://doi org/10.1101/2021.05.27 446087; this version posted May 28,2021 . The copyright holder for this preprint

(which was not certified by peer review) is the author/funder, who has granted bioRxiv a license to display the preprint in perpetuity. It is made available under aCC-BY-NC-ND 4.0 International license.

274.

977 81. Page AJ, Taylor B, Delaney AJ, Soares J, Seemann T, Keane JA, Harris SR. 2016. SNP-sites: 978 rapid efficient extraction of SNPs from multi-FASTA alignments. Microb genomics 2:e000056$979 \quad \mathrm{e} 000056$

980 82. Crispell J, Balaz D, Gordon SV. 2019. HomoplasyFinder: a simple tool to identify homoplasies 981 on a phylogeny. Microb genomics 2019/01/21. 5:e000245.

982 83. Bruen TC, Philippe H, Bryant D. 2006. A simple and robust statistical test for detecting the presence of recombination. Genetics 2006/02/19. 172:2665-2681.

984 84. European Committee on Antimicrobial Susceptibility Testing. 2020. Antimicrobial susceptibility 985 testing EUCAST disk diffusion method Version 8.0.

986 85. Sorlozano-Puerto A, Lopez-Machado I, Albertuz-Crespo M, Martinez-Gonzalez LJ, Gutierrez987 Fernandez J. 2020. Characterization of Fosfomycin and Nitrofurantoin Resistance Mechanisms in Escherichia coli Isolated in Clinical Urine Samples. Antibiotics 9:534. 